Research Article

\title{
Characterization and Antifungal Activity of Limonoid Constituents Isolated from Meliaceae Plants Melia dubia, Aphanamixis polystachya, and Swietenia macrophylla against Plant Pathogenic Fungi In Vitro
}

\author{
Thanh Nguyen Tan $\mathbb{D}^{1},{ }^{1}$ Hieu Tran Trung $\mathbb{D},{ }^{2}$ Quang Le Dang $\mathbb{D},{ }^{3}$ Hien Vu Thi $\mathbb{D}^{4}$, \\ Hoang Dinh Vu $\mathbb{D}^{5},{ }^{5}$ Tuan Nguyen Ngoc $\mathbb{D}^{6},{ }^{6}$ Hoai Thu Thi Do, ${ }^{3}$ Thanh Huong Nguyen, ${ }^{3}$ \\ Dang Ngoc Quang, ${ }^{7}$ and Thang Tran Dinh ${ }^{6}{ }^{6}$ \\ ${ }^{1}$ School of Chemistry Biology and Environment, Vinh University, Vinh, Nghean 43100, Vietnam \\ ${ }^{2}$ School of Natural Sciences Education, Vinh University, Vinh, Nghean 43100, Vietnam \\ ${ }^{3}$ R D Center of Bioactive Compounds, Vietnam Institute of Industrial Chemistry, Hanoi 10000, Vietnam \\ ${ }^{4}$ Ho Chi Minh City University of Natural Resources and Environment, Ho Chi Minh 72114, Vietnam \\ ${ }^{5}$ The School of Chemical Engineering, Hanoi University of Science and Technology, Hanoi 10000, Vietnam \\ ${ }^{6}$ Institute of Biotechnology and Food Technology, Industrial University of Ho Chi Minh City, Ho Chi Minh 71408, Vietnam \\ ${ }^{7}$ Hanoi National University of Education, Hanoi 10000, Vietnam
}

Correspondence should be addressed to Quang Le Dang; ledangquang2011@gmail.com and Thang Tran Dinh; thangtd@iuh.edu.vn

Received 13 May 2021; Accepted 20 July 2021; Published 31 July 2021

Academic Editor: Xinyong Liu

Copyright (c) 2021 Thanh Nguyen Tan et al. This is an open access article distributed under the Creative Commons Attribution License, which permits unrestricted use, distribution, and reproduction in any medium, provided the original work is properly cited.

\begin{abstract}
The plants of Meliaceae are native to tropical and subtropical regions as the Americas, west India, Southeast Asia, and Southern China. Many species of the genera Khaya, Swietenia, Aphanamixis, and Melia in this family are known as medicinal plants and have biological activities such as antiviral, antimicrobial, antifeeding, insecticidal, and cytotoxic properties. The objectives of this research are to characterize and evaluate the bioactive limonoids from several plants of Meliaceae against phytopathogenic fungi. During the search of antifungal compounds from the plants of Meliaceae, the three methanol extracts of Melia dubia, Aphanamixis polystachya, and Swietenia macrophylla were found to suppress the mycelial growth of several phytopathogenic fungi. Nine limonoids isolated from M. dubia (1-2), A. polystachya (3-5), and S. macrophylla (6-9) were evaluated, for the first time, their antifungal effectiveness against nine phytopathogenic fungi Fusarium oxysporum, Magnaporthe oryzae, Sclerotium rolfsii, Rhizoctonia solani, Alternaria spp., and Botrytis cinerea, and three oomycetes Phytophthora species. Limonoids 2, 3, 6, and $\mathbf{8}$ displayed a remarkable broad-spectrum antifungal activity against all the test fungi. Sclerotium rolfsii was highly sensitive to the four limonoids with $\mathrm{IC}_{50}$ values ranging from 79.4 to $128.0 \mu \mathrm{g} / \mathrm{mL}$. Notably, chisocheton compound G (3) isolated from A. polystachya and khayanolide B (8) isolated from S. macrophylla were the most potent antifungal limonoids and exhibited a dose-dependent activity against Phytophthora species. Compounds 2 and 9 displayed moderate activity against $M$. oryzae. Our study results demonstrated the discovery of antifungal and lead compounds from the group of limonoids for application in the control of fungal plant diseases.
\end{abstract}

\section{Introduction}

The Meliaceae family has 50 genera and 1,400 species and distributed in tropical and subtropical regions as the Americas, west India, Southeast Asia, and Southern China
$[1,2]$. Almost all limonoids (more than 300 compounds) have been identified, and about one-third has been found in Azadirachta indica and Melia azedarach [3]. There were more than 160 limonoids isolated from four species of the genus Swietenia [4]. The seeds of S. macrophylla contain 
bioactive compounds with anti-inflammatory, antimutagenicity, and antitumor activity, which has been used in traditional medicine in the world. Furthermore, the seeds of S. macrophylla in Malaysia are used traditionally to treat hypertension, diabetes, and relieve pain [1]. Limonoids and their derivatives are determined as the major constituents of S. macrophylla.

The application of limonoids in plant protection has been developed since the 1960s. To date, most of the Meliaceous limonoids have been reported to be related to antifeedant, insecticidal, cytotoxic, antimalarial, and anticancer activity. Azadirachtin is an insect growth regulator and feeding deterrent that affects more than 60 insects such as aphids, caterpillars, leafhoppers, leafminers, mealybugs, psyllids, thrips, and whiteflies by effects on feeding and reducing ecdysone hormone in insects [5]. Gedunin, nimbin, and nimbolide isolated from Azadirachta indica and K. grandifoliola have been reported to have in vitro antimalarial activity on P. falciparum. According to Roy and Saraf (2006), the $\mathrm{C}$-seco limonoids with an enone system in ring " $\mathrm{A}$ " are potent cytotoxic and antimalarial agents. Moreover, the C-seco limonoids are two to three times more active than other limonoids and they are also highly active against herbivorous insects [3]. The well-known insecticidal limonoids (azadirachtin, salannin, and nimbin) isolated from neem have been used as an active ingredient in a number of commercial insecticide formulations and marketed in many countries [6-8]. Over the years, the well-known limonoids and neem extracts were documented as successful examples of botanical insecticides commercialized. Based on the record of known safety, mode of action, and interaction of the limonoid active ingredients, the chemical class is expected to repurpose or use widely for controlling the phytopathogens in organic agriculture [9-13]. However, to our best knowledge, there are very few studies on the activity of Meliaceous limonoids against phytopathogenic fungi. Several antifungal limonoids against fungal plant pathogens have been studied and reported to occur in the fruits of Khaya senegalensis, K. ivorensis, and Aphanamixis polystachya. Zhang et al. (2013) identified prieurianin-type limonoids and aphanamolide-type limonoids from the fruits of $A$. polystachya and conducted some preliminary experiments to evaluate for their fungicidal, herbicidal, and insecticidal activities [14]. Methyl angolensate and 1,3,7-trideacetylkhivorin isolated from the fruits of $K$. ivorensis displayed a moderate inhibition against the mycelial growth of Botrytis cinerea, at 1000 and $1500 \mu \mathrm{g} / \mathrm{mL}$ [10]. From the fruits of K. senegalensis, Abdelgaleil et al. (2004) also successfully isolated bioactive limonoids such as seneganolide A, 2-acetoxyseneganolide A, and methyl 6-hydroxyangolensate with antifungal effects on B. cinerea [9]. In our ongoing research studies on bioactive limonoids from Meliaceous plants growing in Vietnam, we successfully isolated and identified nine limonoids from Melia dubia (1-2), Aphanamixis polystachya (3-5), and Swietenia macrophylla (6-9). The antifungal potential of the isolated limonoids against various phytopathogenic fungi including $F$. oxysporum, M. oryzae, S. rolfsii, R. solani, Alternaria spp., $B$. cinerea, and oomycetes Phytophthora species was evaluated in vitro.

\section{Materials and Methods}

2.1. Plant Materials. The fruits of three species (Melia dubia, Aphanamixis polystachya, and Swietenia macrophylla) were collected from July to August 2017 from Pu Huong Nature Reserve, Nghe An Province; Vu Quang Nature Reserve, Ha Tinh Province; and Cat Tien National Park, Dong Nai Province, Vietnam, respectively. The plant materials were identified by Dr. Quoc Binh Nguyen, Vietnam National Museum of Nature, Vietnam Academy of Science and Technology, Hanoi, Vietnam. The voucher samples (no. MDF-072017, APF-082017, and SMF-082017) were deposited in the same museum.

2.2. Microbial Strains and Culture Conditions. The in vitro antifungal activity of the botanical extracts and purified limonoids was tested based on poisoned food technique against nine phytopathogenic fungi including Fusarium oxysporum, Magnaporthe oryzae, Sclerotium rolfsii, Rhizoctonia solani, Botrytis cinerea, Alternaria spp., Phytophthora capsici, Phytophthora palmivora, and Phytophthora spp. Out of oomycetes Phytophthora species, Phytophthora spp. was isolated from the infected roots of Panax vietnamensis $\mathrm{Ha}$ et Grushv., and P. palmivora and P. capsici were isolated from the infected leaves of durian (Durio zibethinus) and black pepper (Piper nigrum), respectively. Potato dextrose broth (PDB) was used to subculture, and the potato dextrose agar (PDA) medium was used to culture fungal strains for antifungal tests at $20-25^{\circ} \mathrm{C}$ for $2-7$ days.

2.3. Isolation and Characterization of Phytochemical Constituents from Melia dubia, Aphanamixis polystachya, and Swietenia macrophylla. The dried fruit powder of Melia dubia $(16.0 \mathrm{~kg})$ was extracted with methanol at ambient temperature ( $20 \mathrm{~L}, 5$ times), and total methanol extract was evaporated under reduced pressure to give the methanol crude extract $(854 \mathrm{~g})$. Then, it was suspended in water and partitioned successively with $n$-hexane, ethyl acetate, and butanol to afford $n$-hexane extract (MDH-68 g), ethyl acetate extract (MDE-272 g), butanol extract (MDB-133g), and water-soluble fraction $(80 \mathrm{~g})$, respectively. The ethyl acetate extract $(272 \mathrm{~g})$ was applied to silica gel column chromatography and was eluted by a mixture of chloroform/ methanol with gradient $(100: 0,50: 1,30: 1,20: 1,10: 1,5: 1$, $2: 1$, and $1: 1$ ) to afford ten fractions (Frs. F1-F10). Fraction F2 $(16.5 \mathrm{~g})$ was subjected to silica gel column chromatography $(150 \mathrm{~g}, 80 \times 2 \mathrm{~cm})$ eluting by a mixture of $n$-hexane/ acetone with gradient $15: 1$ to obtain six fractions (F2.1-F2.6). Fraction F2.1 (2.5 g) was subjected to the silica gel column chromatography $(300 \mathrm{~g}, 80 \times 3 \mathrm{~cm})$ eluting with a mixture of $n$-hexane: acetone $(7: 1)$ to give compound 1 (MD1, $21 \mathrm{mg})$. Fraction F2.2 (0.2 g) was separated by preparative HPLC $\left(\mathrm{MeOH} / \mathrm{H}_{2} \mathrm{O}, 80: 40\right.$ to $90: 10,10 \mathrm{~min}, 16 \mathrm{ml}$ $\mathrm{min}^{-1}$ ) to afford compound 2 (MD2, $12 \mathrm{mg}$ ) (Figure S1a).

The dried fruit powder of Aphanamixis polystachya $(9.0 \mathrm{~kg})$ was extracted with methanol at ambient temperature (10 L, 3 times), and total methanol extract was evaporated under reduced pressure to give the methanol crude extract 
$(480 \mathrm{~g})$. Then, it was suspended in water and partitioned successively with $n$-hexane, ethyl acetate, and butanol to afford $n$-hexane extract (APH, $68 \mathrm{~g})$, ethyl acetate extract (APE, $172 \mathrm{~g}$ ), butanol extract (APB, $87 \mathrm{~g}$ ), and water-soluble fraction (APW, $100 \mathrm{~g}$ ), respectively. The ethyl acetate extract $(172 \mathrm{~g})$ was applied to silica gel column chromatography and was eluted by a mixture of chloroform/methanol with gradient $(100: 0,50: 1,30: 1,20: 1,10: 1,5: 1,2: 1$, and $1: 1)$ to afford seven fractions (F1-F7). Fraction F3 (5.9g) was subjected to silica gel column chromatography (120 g, $80 \times 1.5 \mathrm{~cm}$ ) eluting by a mixture of $n$-hexane/ethyl acetate with gradient 9:1 to obtain five fractions (Frs. F2.1-F2.5). Fraction F3.2 (1.68 g) was subjected to the silica gel column chromatography $(150 \mathrm{~g}, 80 \times 2 \mathrm{~cm})$ eluting with a mixture of $n$-hexane: ethyl acetate $(5: 1)$ to give compound 3 (AP1, $11 \mathrm{mg})$. Fraction F3.3 (0.2 g) was separated by the silica gel column chromatography ( $n$-hexane: ethyl acetate, $20: 10$ to 20:5) to afford compound 4 (AP2, $21 \mathrm{mg}$ ) and compound 5 (AP3, $14 \mathrm{mg}$ ) (Figure S1b).

The dried powdered fruits of Swietenia macrophylla $(6.0 \mathrm{~kg})$ were extracted with methanol $(3$ times $\times 10 \mathrm{~L})$ at room temperature to yield $318.0 \mathrm{~g}$ of the crude methanol extract. The crude extract was then suspended in distillate water and introduced to liquid-liquid extraction partitioning in turn with $n$-hexane and ethyl acetate to obtain crude $n$ hexane and ethyl acetate, respectively. The ethyl acetate extract $(105.0 \mathrm{~g})$ was chromatographed by a silica gel column and eluted with a $n$-hexane: ethyl acetate (gradient 100:1 to $1: 1)$ to yield 5 fractions. Fraction 4 was separated by a silica gel column, eluting with $n$-hexane-ethyl acetate (25:1 to 2 : 1) to give 7 subfractions from 4.1 to 4.7 . Fraction 4.3 was rechromatographed by a silica column eluted with $n$-hexane: ethyl acetate (15:1) to afford compound 7 (SM2, $13 \mathrm{mg}$ ). Then, fraction 4.4 was rechromatographed by a silica column eluted with $n$-hexane: ethyl acetate $(9: 1)$ to yield compounds 6 (SM1, $18 \mathrm{mg}$ ) and 8 (SM3, 34 mg) were isolated from fraction 4.5 by using a silica gel column and eluted $n$-hexane: ethyl acetate $(9: 1 ; 4: 1)$. Compound 9 (SM4, 12.5 mg) (Figure S1c) was obtained from the purification of fraction 5 by silica gel column chromatography eluting with $\mathrm{CH}_{2} \mathrm{Cl}_{2}$ : $\mathrm{CH}_{3} \mathrm{OH}(9: 1)$.

\subsection{Structural Characterization of the Isolated Compounds}

2.4.1. Compound 1 (MD1): Dysobinin [15, 16]. Colorless crystals, m.p. $209-210^{\circ} \mathrm{C}$; HR-ESI-MS $\mathrm{m} / \mathrm{z} 517.2629$ $[\mathrm{M}+\mathrm{Na}]^{+} ;{ }^{1} \mathrm{H}$ NMR (DMSO- $\left.d_{6}, 500 \mathrm{MHz}\right): d(\mathrm{ppm}): 7.38(t$, $\left.J=3.5 \mathrm{~Hz}, \mathrm{C}_{1}-\mathrm{H}\right), 7.24\left(s, \mathrm{C}_{21}-\mathrm{H}\right), 7.14(d d, J=2.5,10.5 \mathrm{~Hz}$, $\left.\mathrm{C}_{23}-\mathrm{H}\right), 6.28\left(s, \mathrm{C}_{22}-\mathrm{H}\right), 5.92\left(d d, J=3.5,10.0 \mathrm{~Hz}, \mathrm{C}_{2}-\mathrm{H}\right), 5.46$ $\left(d, J=3.0 \mathrm{~Hz}, \mathrm{C}_{7}-\mathrm{H}\right), 5.43\left(m, \mathrm{C}_{6}-\mathrm{H}\right), 5.39\left(s, \mathrm{C}_{15}-\mathrm{H}\right), 2.82(m$, $\left.\mathrm{C}_{17}-\mathrm{H}\right), 2.51\left(d d, J=3.0,11.5 \mathrm{~Hz}, \mathrm{C}_{5}-\mathrm{H}\right), 2.04\left(s, \mathrm{C}_{7^{-}}\right.$ $\left.\mathrm{OCOCH}_{3}\right), 2.02\left(s, \mathrm{C}_{6}-\mathrm{OCOCH}_{3}\right), 1.33\left(s, \mathrm{C}_{19}-\mathrm{H}\right), 1.26(s$, $\left.\mathrm{C}_{30}-\mathrm{H}\right), 1.19\left(s, \mathrm{C}_{28,29}-\mathrm{H}\right)$, and $0.81\left(m, \mathrm{C}_{18}-\mathrm{H}\right) ;{ }^{13} \mathrm{C} \mathrm{NMR}$ (DMSO- $\left.d_{6}, 125 \mathrm{MHz}\right): d(\mathrm{ppm}): \mathrm{C}_{3}(204.6), 6-\mathrm{OCO}(170.2)$, 7-OCO (170.0), C $\mathrm{C}_{14}$ (158.2), C $\mathrm{C}_{1}$ (157.2), $\mathrm{C}_{21}$ (142.5), $\mathrm{C}_{23}$ (139.6), $\mathrm{C}_{2}$ (126.1), $\mathrm{C}_{20}$ (124.3), $\mathrm{C}_{15}$ (119.7), $\mathrm{C}_{22}$ (110.9), $\mathrm{C}_{7}$ (74.5), $\mathrm{C}_{6}$ (69.9), $\mathrm{C}_{17}$ (51.6), $\mathrm{C}_{5}(47.9), \mathrm{C}_{13}(47.06), \mathrm{C}_{8}$ (44.8), $\mathrm{C}_{10}$ (43.0), $\mathrm{C}_{4}(40.7), \mathrm{C}_{9}(37.3), \mathrm{C}_{16}(34.3), \mathrm{C}_{12}(32.7), \mathrm{C}_{18}$
(31.5), $\mathrm{C}_{28}$ (26.7), 7- $-\mathrm{OCOCH}_{3}$ (21.3), 6- $\mathrm{OCOCH}_{3}(21.0), \mathrm{C}_{29}$ (20.8), $\mathrm{C}_{30}$ (20.7), $\mathrm{C}_{19}(20.3)$, and $\mathrm{C}_{11}$ (16.3).

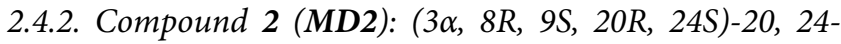
Epoxytirucalla-3, 25-Diol [17]. White crystals, m.p. 236-237 ${ }^{\circ}$; HR-ESI-MS $\mathrm{m} / z$ 461.3986 $[\mathrm{M}+\mathrm{H}]^{+}$; ${ }^{1} \mathrm{H}$ NMR $\left(\mathrm{CDCl}_{3}, 500 \mathrm{MHz}\right): \delta(\mathrm{ppm}): 3.73\left(t, J=7.0 \mathrm{~Hz}, \mathrm{C}_{24}-\mathrm{H}\right), 3.39$ $\left(t, J=2.5 \mathrm{~Hz}, \mathrm{C}_{3}-\mathrm{H}\right), 1.94\left(m, \mathrm{C}_{2}-\mathrm{H}\right), 1.86\left(m, \mathrm{C}_{22}-\mathrm{H}\right), 1.71$ $\left(m, \mathrm{C}_{7}-\mathrm{H}\right), 1.62\left(m, \mathrm{C}_{7}-\mathrm{H}\right), 1.58\left(m, \mathrm{C}_{16}-\mathrm{H}\right), 1.56\left(m, \mathrm{C}_{11}-\mathrm{H}\right)$, $1.55\left(m, \mathrm{C}_{8}-\mathrm{H}\right), 1.52\left(m, \mathrm{C}_{2}-\mathrm{H}\right), 1.45\left(m, \mathrm{C}_{6,9}-\mathrm{H}\right), 1.44(m$, $\left.\mathrm{C}_{22}-\mathrm{H}\right), 1.42\left(m, \mathrm{C}_{15}-\mathrm{H}\right), 1.41\left(m, \mathrm{C}_{1,23}-\mathrm{H}\right), 1.27\left(m, \mathrm{C}_{1}-\mathrm{H}\right)$, $1.26\left(m, \mathrm{C}_{6}-\mathrm{H}\right), 1.25\left(m, \mathrm{C}_{5,21}-\mathrm{H}\right), 1.24\left(m, \mathrm{C}_{23}-\mathrm{H}\right), 1.20$ $\left(s, \mathrm{C}_{27}-\mathrm{H}\right), 1.17\left(m, \mathrm{C}_{11}-\mathrm{H}\right), 1.12\left(d, J=5.5 \mathrm{~Hz}, \mathrm{C}_{12,26}-\mathrm{H}\right), 1.07$ $\left(t, J=17.5 \mathrm{~Hz}, \mathrm{C}_{15}-\mathrm{H}\right), 0.96\left(s, \mathrm{C}_{30}-\mathrm{H}\right), 0.94\left(s, \mathrm{C}_{28}-\mathrm{H}\right), 0.88$ $\left(s, \mathrm{C}_{18}-\mathrm{H}\right), 0.85\left(s, \mathrm{C}_{19}-\mathrm{H}\right)$, and $0.84\left(s, \mathrm{C}_{29}-\mathrm{H}\right) ;{ }^{13} \mathrm{C}$ NMR $\left(\mathrm{CDCl}_{3}, 125 \mathrm{MHz}\right): \delta(\mathrm{ppm}): \mathrm{C}_{20}(86.4), \mathrm{C}_{24}(83.3), \mathrm{C}_{3}(76.3)$, $\mathrm{C}_{25}$ (71.5), $\mathrm{C}_{9}$ (50.6), $\mathrm{C}_{14}(50.2), \mathrm{C}_{5,17}$ (49.6), $\mathrm{C}_{8}(42.9), \mathrm{C}_{13}$ (40.6), $C_{4}$ (37.6), $C_{10}$ (37.3), $C_{7}(35.7), C_{23}$ (35.2), $C_{1}$ (33.7), $\mathrm{C}_{15}$ (31.4), $\mathrm{C}_{28}$ (28.3), $\mathrm{C}_{21,27}$ (27.4), $\mathrm{C}_{12,22}$ (26.2), $\mathrm{C}_{2}$ (25.7), $\mathrm{C}_{16}$ (25.4), $\mathrm{C}_{26}$ (24.3), $\mathrm{C}_{29}$ (22.1), $\mathrm{C}_{11}$ (21.4), $\mathrm{C}_{6}(18.2), \mathrm{C}_{18}$ (16.6), $\mathrm{C}_{19}$ (16.0), and $\mathrm{C}_{30}$ (15.5).

2.4.3. Compound 3 (AP1): Chisocheton Compound G [18]. White powder, m.p. $182-183^{\circ} \mathrm{C}$; HR-ESI-MS $\mathrm{m} / z$ 549.2566 $[\mathrm{M}+\mathrm{Na}]^{+} ;{ }^{1} \mathrm{H}$ NMR $\left(\mathrm{DMSO}-d_{6}, 500 \mathrm{MHz}\right): \delta(\mathrm{ppm}): 7.17$ $\left(m, \mathrm{C}_{1}-\mathrm{H}\right), 5.98\left(s, \mathrm{C}_{21}-\mathrm{H}\right), 5.93\left(d, J=10.5 \mathrm{~Hz}, \mathrm{C}_{2}-\mathrm{H}\right), 5.90(s$, $\left.\mathrm{C}_{22}-\mathrm{H}\right), 5.45\left(d, J=3.0 \mathrm{~Hz}, \mathrm{C}_{7}-\mathrm{H}\right), 5.42\left(d, J=3.5 \mathrm{~Hz}, \mathrm{C}_{6}-\mathrm{H}\right)$, $5.40\left(d, J=2.5 \mathrm{~Hz}, \mathrm{C}_{15}-\mathrm{H}\right), 2.52\left(s, \mathrm{C}_{5}-\mathrm{H}\right), 2.05\left(s, \mathrm{C}_{7^{-}}\right.$ $\left.\mathrm{OCOCH}_{3}\right), 2.02\left(s, \mathrm{C}_{6}-\mathrm{OCOCH}_{3}\right), 1.33\left(s, \mathrm{C}_{19}-\mathrm{H}\right), 1.26(s$, $\left.\mathrm{C}_{30}-\mathrm{H}\right), 1.19\left(s, \mathrm{C}_{28,29}-\mathrm{H}\right)$, and $0.93\left(m, \mathrm{C}_{18}-\mathrm{H}\right) .{ }^{13} \mathrm{C}$ NMR (DMSO- $\left.d_{6}, 125 \mathrm{MHz}\right): \delta$ (ppm): $\mathrm{C}_{3}(204.9), \mathrm{C}_{23}(171.6), \mathrm{C}_{20}$ (170.4), 6-OCO (170.2), 7-OCO (169.5), $\mathrm{C}_{14}$ (157.5), $\mathrm{C}_{1}$ (157.2), $\mathrm{C}_{2}$ (126.1), $\mathrm{C}_{15}$ (119.6), $\mathrm{C}_{22}$ (118.6), $\mathrm{C}_{21}$ (99.1), $\mathrm{C}_{7}$ (74.5), $\mathrm{C}_{6}$ (69.8), $\mathrm{C}_{17}$ (52.7), $\mathrm{C}_{5}(47.8), \mathrm{C}_{13}$ (47.2), $\mathrm{C}_{10}$ (44.9), $\mathrm{C}_{8}$ (43.1), $\mathrm{C}_{4}$ (40.7), $\mathrm{C}_{9}(37.1), \mathrm{C}_{12}$ (33.2), $\mathrm{C}_{16}$ (33.0), $\mathrm{C}_{18}$ (31.5), $\mathrm{C}_{28}$ (26.6), 7-OCOCH 3 (21.4), 6- $\mathrm{OCOCH}_{3}(21.1), \mathrm{C}_{29}$ (20.8), $\mathrm{C}_{30}(20.6), \mathrm{C}_{19}(20.2)$, and $\mathrm{C}_{11}(16.3)$.

2.4.4. Compound 4 (AP2): Chisocheton Compound E [18]. Colorless crystals, m.p. $236-237^{\circ} \mathrm{C}$; ESI-MS $\mathrm{m} / \mathrm{z} 513.2$ $[\mathrm{M}+\mathrm{H}]^{+} ;{ }^{1} \mathrm{H}$ NMR (DMSO- $\left.d_{6}, 500 \mathrm{MHz}\right): \delta(\mathrm{ppm}): 7.13(d$, $\left.J=10.0 \mathrm{~Hz}, \mathrm{C}_{1}-\mathrm{H}\right), 5.95\left(d, J=10.0 \mathrm{~Hz}, \mathrm{C}_{2}-\mathrm{H}\right), 5.45(d$, $\left.J=3.5 \mathrm{~Hz}, \mathrm{C}_{7}-\mathrm{H}\right), 5.42\left(d, J=6.0 \mathrm{~Hz}, \mathrm{C}_{6}-\mathrm{H}\right), 5.40(d, J=2.5 \mathrm{~Hz}$, $\left.\mathrm{C}_{15}-\mathrm{H}\right), 4.49\left(t, J=3.0 \mathrm{~Hz}, \mathrm{C}_{21}-\mathrm{H}_{\mathrm{a}}\right), 3.94\left(t, J=4.5 \mathrm{~Hz}, \mathrm{C}_{21}-\mathrm{H}_{\mathrm{b}}\right)$, $2.75\left(m, \mathrm{C}_{20}-\mathrm{H}\right), 2.52\left(s, \mathrm{C}_{5}-\mathrm{H}\right), 2.04\left(s, \mathrm{C}_{7}-\mathrm{OCOCH}_{3}\right), 1.99(s$, $\left.\mathrm{C}_{6}-\mathrm{OCOCH}_{3}\right), 1.29\left(s, \mathrm{C}_{19^{-}} \mathrm{H}\right), 1.26\left(s, \mathrm{C}_{30}-\mathrm{H}\right), 1.18\left(s, \mathrm{C}_{28,29^{-}}\right.$ $\mathrm{H})$, and $1.04\left(m, \mathrm{C}_{18}-\mathrm{H}\right) \cdot{ }^{13} \mathrm{C}$ NMR (DMSO- $\left.d_{6}, 125 \mathrm{MHz}\right): d$ (ppm): $\mathrm{C}_{3}$ (204.4), $\mathrm{C}_{23}$ (176.4), 6-OCO (170.2), 7-OCO (169.9), $\mathrm{C}_{14}$ (158.0), $\mathrm{C}_{1}$ (156.8), $\mathrm{C}_{2}$ (126.3), $\mathrm{C}_{15}$ (119.6), $\mathrm{C}_{7}$ (74.4), $\mathrm{C}_{21}$ (72.3), $\mathrm{C}_{6}$ (69.8), $\mathrm{C}_{17}(58.2), \mathrm{C}_{5}$ (48.0), $\mathrm{C}_{13}$ (46.6), $\mathrm{C}_{10}$ (44.9), $\mathrm{C}_{8}(42.9), \mathrm{C}_{4}(40.8), \mathrm{C}_{9,20}$ (37.0), $\mathrm{C}_{22}(34.8), \mathrm{C}_{12,16}$ (33.5), $\mathrm{C}_{18}$ (31.7), $\mathrm{C}_{28}$ (26.9), 7- $-\mathrm{OCOCH}_{3}$ (21.3), 6- $-\mathrm{OCOCH}_{3}$ (20.9), $\mathrm{C}_{29}(20.7), \mathrm{C}_{30}(20.4), \mathrm{C}_{19}(20.1)$, and $\mathrm{C}_{11}(16.4)$.

2.4.5. Compound 5 (AP3): $6 \alpha$-Acetoxyepoxyazadiradione VI [19]. Colorless crystals, m.p. $167-168^{\circ} \mathrm{C}$; ESI-MS $m / z 525.1$ $[\mathrm{M}+\mathrm{H}]^{+} ;{ }^{1} \mathrm{H}$ NMR (DMSO- $\left.d_{6}, 500 \mathrm{MHz}\right): \delta(\mathrm{ppm}): 7.55$ 
$\left(\mathrm{C}_{21}-\mathrm{H}\right), 7.4\left(\mathrm{C}_{23}-\mathrm{H}\right), 7,12\left(d, J=10 \mathrm{~Hz}, \mathrm{C}_{1}-\mathrm{H}\right), 6.23\left(\mathrm{C}_{22}-\mathrm{H}\right)$, $5.94\left(d, J=10.5 \mathrm{~Hz}, \mathrm{C}_{2}-\mathrm{H}\right), 5.37\left(d d, J=12.5,2.5 \mathrm{~Hz}, \mathrm{C}_{6}-\mathrm{H}\right)$, $5.03\left(d, J=2.5 \mathrm{~Hz}, \mathrm{C}_{7}-\mathrm{H}\right), 3.89\left(s, \mathrm{C}_{17}-\mathrm{H}\right), 3.43\left(s, \mathrm{C}_{15}-\mathrm{H}\right)$, $2.53\left(m, \mathrm{C}_{9}-\mathrm{H}\right), 2.52\left(d, J=12.5 \mathrm{~Hz}, \mathrm{C}_{5}-\mathrm{H}\right), 2.08\left(s, \mathrm{C}_{7^{-}}\right.$ $\left.\mathrm{OCOCH}_{3}\right), 2.03\left(s, \mathrm{C}_{6}-\mathrm{OCOCH}_{3}\right), 1.93\left(m, \mathrm{C}_{11}-\mathrm{H}_{\mathrm{a}}\right), 1.84(m$, $\left.\mathrm{C}_{11}-\mathrm{H}_{\mathrm{b}}\right), 1.32\left(\mathrm{C}_{26}-\mathrm{H}\right), 1.25\left(\mathrm{C}_{24}-\mathrm{H}\right), 1.21\left(s, \mathrm{C}_{18,19}-\mathrm{H}\right)$, and $1.04\left(\mathrm{C}_{25}-\mathrm{H}\right) .{ }^{13} \mathrm{C}$ NMR (DMSO- $\left.d_{6}, 125 \mathrm{MHz}\right): \delta(\mathrm{ppm}): \mathrm{C}_{3}$ (204.1), 6- $-\mathrm{OCOCH}_{3}$ (169.8), 7- $\mathrm{OCOCH}_{3}$ (169.7), $\mathrm{C}_{1,16}$ (156.6), $\mathrm{C}_{23}$ (142.4), $\mathrm{C}_{21}$ (141.5), $\mathrm{C}_{2,20}$ (126.4), $\mathrm{C}_{22}$ (110.8), $\mathrm{C}_{7}$ (72.1), $\mathrm{C}_{6,14}$ (69.7), $\mathrm{C}_{15}$ (57.0), $\mathrm{C}_{13,17}$ (50.7), $\mathrm{C}_{9}$ (48.5), $\mathrm{C}_{5}$ (48.4), $\mathrm{C}_{4}(45.0), \mathrm{C}_{8}(43.2), \mathrm{C}_{10}(40.4), \mathrm{C}_{24}$ (31.5), $\mathrm{C}_{12}$ (24.5), $\mathrm{C}_{19}$ (21.4), 6,7-OCOCH${ }_{3}(21.1), \mathrm{C}_{25,26}(20.1), \mathrm{C}_{18}$ (18.9), and $\mathrm{C}_{11}(16.0)$.

2.4.6. Compound 6 (SM1): Seneganolide [20]. White powder, m.p. $276-277^{\circ} \mathrm{C}$; $\quad$ ESI-MS m/z $471.1 \quad[\mathrm{M}+\mathrm{H}]^{+}$ $\left(\mathrm{C}_{26} \mathrm{H}_{31} \mathrm{O}_{8}\right) ;{ }^{1} \mathrm{H}$ NMR $\left(\mathrm{CDCl}_{3}\right.$ and $\left.\mathrm{CD}_{3} \mathrm{OD}, 500 \mathrm{MHz}\right): \delta$ (ppm): $7.40\left(d, J=2.0 \mathrm{~Hz}, \mathrm{C}_{23}-\mathrm{H}\right), 7.40$ (brs, $\left.\mathrm{C}_{21}-\mathrm{H}\right), 6.33$ $\left(t, J=1.0 \mathrm{~Hz}, \mathrm{C}_{22}-\mathrm{H}\right), 5.28\left(s, \mathrm{C}_{17}-\mathrm{H}\right), 4.45(d, J=11.5 \mathrm{~Hz}$, $\left.\mathrm{C}_{19}-\mathrm{H}_{\alpha}\right), 4.21\left(d, J=12.0 \mathrm{~Hz}, \mathrm{C}_{19}-\mathrm{H}_{\beta}\right), 2.84(d d, J=7.0$, $\left.13.5 \mathrm{~Hz}, \mathrm{C}_{2}-\mathrm{H}\right), 2.83\left(d d, J=7.0,19.5 \mathrm{~Hz}, \mathrm{C}_{15}-\mathrm{H}_{\beta}\right), 2.78(d d$, $\left.J=2.0,19.5 \mathrm{~Hz}, \mathrm{C}_{15}-\mathrm{H}_{\alpha}\right), 2.70\left(d d, J=11.0,15.0 \mathrm{~Hz}, \mathrm{C}_{6}-\mathrm{H}_{\alpha}\right)$, $2.56\left(d d, J=7.0,15.0 \mathrm{~Hz}, \mathrm{C}_{6}-\mathrm{H}_{\beta}\right), 2.32(d d, J=10.5,7.0 \mathrm{~Hz}$, $\left.\mathrm{C}_{5} \mathrm{H}\right), 2.27\left(d d, J=2.0,7.0 \mathrm{~Hz}, \mathrm{C}_{14}-\mathrm{H}\right), 2.20(t d, J=2.0$, $\left.13.5 \mathrm{~Hz}, \mathrm{C}_{30}-\mathrm{H}_{\alpha}\right), 2.01\left(b r d, J=11.5 \mathrm{~Hz}, \mathrm{C}_{9}-\mathrm{H}\right), 1.87$ (dd, $\left.J=7.0,13.5 \mathrm{~Hz}, \mathrm{C}_{30}-\mathrm{H}_{\beta}\right), 1.76\left(d d, J=2.0,11.0 \mathrm{~Hz}, \mathrm{C}_{12}-\mathrm{H}_{\beta}\right)$, $1.61\left(m, \mathrm{C}_{11}-\mathrm{H}_{\alpha}\right), 1.41\left(m, \mathrm{C}_{11}-\mathrm{H}_{\beta}\right), 1.41(b r t, J=12.5 \mathrm{~Hz}$, $\left.\mathrm{C}_{12}-\mathrm{H}_{\alpha}\right), 1.32\left(s, \mathrm{C}_{29}-\mathrm{H}\right), 1.19\left(s, \mathrm{C}_{28}-\mathrm{H}\right)$, and $1.03\left(s, \mathrm{C}_{18}-\mathrm{H}\right)$. ${ }^{13} \mathrm{C} \mathrm{NMR}\left(\mathrm{CDCl}_{3} \& \mathrm{CD}_{3} \mathrm{OD}, 125 \mathrm{MHz}\right): d(\mathrm{ppm}): \mathrm{C}_{3}(213.5)$, $\mathrm{C}_{7}$ (174.1), $\mathrm{C}_{16}$ (170.6), $\mathrm{C}_{23}$ (143.1), $\mathrm{C}_{21}$ (140.9), $\mathrm{C}_{20}$ (120.7), $\mathrm{C}_{22}$ (109.6), $\mathrm{C}_{1}$ (107.5), $\mathrm{C}_{8}$ (80.1), $\mathrm{C}_{17}$ (78.1), $\mathrm{C}_{19}$ (74.1), $\mathrm{C}_{9}$ (61.2), $\mathrm{C}_{2}$ (53.0), $\mathrm{C}_{10}$ (46.8), $\mathrm{C}_{14}$ (44.7), $\mathrm{C}_{4}$ (44.7), $\mathrm{C}_{5}$ (38.5), $\mathrm{C}_{13}$ (35.3), $\mathrm{C}_{12}$ (34.9), $\mathrm{C}_{30}$ (31.4), $\mathrm{C}_{6}$ (29.5), $\mathrm{C}_{15}$ (27.5), $\mathrm{C}_{28}$ (23.8), $\mathrm{C}_{18}(22.4), \mathrm{C}_{11}(20.8)$, and $\mathrm{C}_{29}$ (19.6).

2.4.7. Compound 7 (SM2): Khayanone [21]. Colorless crystals, m.p. $170-171^{\circ} \mathrm{C}$; ESI-MS m/z $503.1[\mathrm{M}+\mathrm{H}]^{+}$ $\left(\mathrm{C}_{27} \mathrm{H}_{35} \mathrm{O}_{9}\right) ;{ }^{1} \mathrm{H}$ NMR (DMSO- $\left.d_{6}, 500 \mathrm{MHz}\right): \delta$ (ppm): 7.45 (brs, $\left.\mathrm{C}_{21}-\mathrm{H}\right), 7.43\left(t, J=2.0 \mathrm{~Hz}, \mathrm{C}_{23}-\mathrm{H}\right), 6.37\left(m, \mathrm{C}_{22}-\mathrm{H}\right), 5.60$ $\left(s, \mathrm{C}_{17}-\mathrm{H}\right), 4.42\left(m, \mathrm{C}_{6}-\mathrm{H}\right), 3.83\left(s, 7-\mathrm{COOCH}_{3}\right), 3.14(d$, $\left.J=9.0 \mathrm{~Hz}, \mathrm{C}_{2}-\mathrm{H}\right), 3.13\left(d, J=15.0 \mathrm{~Hz}, \mathrm{C}_{30^{-}}-\mathrm{H}_{\beta}\right), 2.89$ (brs, $\mathrm{C}_{6^{-}}$ $\mathrm{OH}), 2.82\left(d d, J=19.0,2.0 \mathrm{~Hz}, \mathrm{C}_{15}-\mathrm{H}_{\alpha}\right), 2.78$ (brs, $\left.\mathrm{C}_{8}-\mathrm{OH}\right)$, $2.78\left(m, \mathrm{C}_{5}-\mathrm{H}\right), 2.75\left(d d, J=19.0,7.5 \mathrm{~Hz}, \mathrm{C}_{15}-\mathrm{H}_{\beta}\right), 2.36(d d d$, $\left.J=15.0,9.5,2.0 \mathrm{~Hz}, \mathrm{C}_{30}-\mathrm{H}_{\alpha}\right), 1.87\left(d d, J=13.0,5.0 \mathrm{~Hz}, \mathrm{C}_{9^{-}}\right.$ $\mathrm{H}), 1.82\left(m, \mathrm{C}_{11}-\mathrm{H}_{\alpha}\right), 1.75\left(d d, J=7.5,2.0 \mathrm{~Hz}, \mathrm{C}_{14}-\mathrm{H}\right), 1.72$ $\left(m, \mathrm{C}_{12}-\mathrm{H}_{\alpha}\right), 1.36\left(s, \mathrm{C}_{19}-\mathrm{H}\right), 1.28\left(s, \mathrm{C}_{29}-\mathrm{H}\right), 1.27\left(s, \mathrm{C}_{28}-\mathrm{H}\right)$, $1.26\left(m, \mathrm{C}_{12}-\mathrm{H}_{\beta}\right), 1.19\left(m, \mathrm{C}_{11}-\mathrm{H}_{\beta}\right)$, and $0.99\left(s, \mathrm{C}_{18}-\mathrm{H}\right) .{ }^{13} \mathrm{C}$ NMR (DMSO- $\left.d_{6}, 125 \mathrm{MHz}\right): \delta(\mathrm{ppm}): \mathrm{C}_{3}(214.2), \mathrm{C}_{1}(213.1)$, $\mathrm{C}_{7}$ (175.5), $\mathrm{C}_{16}$ (171.2), $\mathrm{C}_{23}$ (143.1), $\mathrm{C}_{21}$ (141.1), $\mathrm{C}_{20}$ (120.9), $\mathrm{C}_{22}$ (109.8), $\mathrm{C}_{17}$ (76.8), $\mathrm{C}_{8}$ (72.9), $\mathrm{C}_{6}$ (70.7), $\mathrm{C}_{9}$ (61.2), $\mathrm{C}_{2}$ (54.3), 7-COOC (53.0), $\mathrm{C}_{14}$ (51.1), $\mathrm{C}_{10}$ (50.2), $\mathrm{C}_{4}$ (50.2), $\mathrm{C}_{5}$ (46.0), $\mathrm{C}_{30}$ (39.0), $\mathrm{C}_{13}$ (35.4), $\mathrm{C}_{12}$ (35.0), $\mathrm{C}_{15}$ (27.1), $\mathrm{C}_{29}$ (26.7), $\mathrm{C}_{19}$ (25.5), $\mathrm{C}_{28}$ (23.8), $\mathrm{C}_{18}$ (23.8), and $\mathrm{C}_{11}$ (22.6).

2.4.8. Compound 8 (SM3): Khayanolide B [22]. Colorless crystals, m.p. $303-304^{\circ} \mathrm{C}$; ESI-MS m/z $519.2[\mathrm{M}+\mathrm{H}]^{+}$ $\left(\mathrm{C}_{27} \mathrm{H}_{35} \mathrm{O}_{10}\right) ;{ }^{1} \mathrm{H}$ NMR (DMSO- $\left.d_{6}, 500 \mathrm{MHz}\right): \delta$ (ppm): 7.47 (brs, $\left.\mathrm{C}_{21}-\mathrm{H}\right), 7.41\left(t, J=2.0 \mathrm{~Hz}, \mathrm{C}_{23}-\mathrm{H}\right), 6.42\left(m, \mathrm{C}_{22}-\mathrm{H}\right), 5.64$ $\left(s, \mathrm{C}_{17}-\mathrm{H}\right), 4.50\left(d d, J=9.0,7.0 \mathrm{~Hz}, \mathrm{C}_{2}-\mathrm{H}\right), 4.20(d, J=7.0 \mathrm{~Hz}$, $\left.\mathrm{C}_{6}-\mathrm{H}_{\alpha}\right), 3.71\left(s, 7-\mathrm{COOCH}_{3}\right), 3.40\left(d, J=7.0 \mathrm{~Hz}, \mathrm{C}_{3}-\mathrm{H}\right), 3.16$ $\left(d, J=19.0 \mathrm{~Hz}, \mathrm{C}_{15}-\mathrm{H}_{\alpha}\right), 3.06\left(d, J=7.0 \mathrm{~Hz}, \mathrm{C}_{5}-\mathrm{H}\right), 2.77(d$, $\left.J=19.0 \mathrm{~Hz}, \mathrm{C}_{15}-\mathrm{H}_{\beta}\right), 2.60\left(d, J=9.5 \mathrm{~Hz}, \mathrm{C}_{30}-\mathrm{H}\right), 2.09(d$, $\left.J=8.0 \mathrm{~Hz}, \mathrm{C}_{9}-\mathrm{H}\right), 1.89\left(d, J=11.5 \mathrm{~Hz}, \mathrm{C}_{29}-\mathrm{H}_{\alpha}\right), 1.86\left(m, \mathrm{C}_{11^{-}}\right.$ $\left.\mathrm{H}_{\alpha}\right), 1.85\left(m, \mathrm{C}_{12}-\mathrm{H}_{\beta}\right), 1.77\left(m, \mathrm{C}_{11}-\mathrm{H}_{\alpha}\right), 1.38(d, J=11.5 \mathrm{~Hz}$, $\left.\mathrm{C}_{29}-\mathrm{H}_{\beta}\right), 1.20\left(s, \mathrm{C}_{19}-\mathrm{H}\right), 1.10\left(s, \mathrm{C}_{18}-\mathrm{H}\right), 1.07\left(q, \mathrm{C}_{28}-\mathrm{H}\right)$, and $0.96\left(m, \mathrm{C}_{12}-\mathrm{H}_{\alpha}\right) .{ }^{13} \mathrm{C}$ NMR (DMSO- $\left.d_{6}, 125 \mathrm{MHz}\right): \delta(\mathrm{ppm})$ : $\mathrm{C}_{7}$ (175.4), $\mathrm{C}_{16}$ (171.7), $\mathrm{C}_{21}$ (140.9), $\mathrm{C}_{23}$ (142.6), $\mathrm{C}_{20}$ (120.6), $\mathrm{C}_{22}$ (110.0), $\mathrm{C}_{8}$ (86.9), $\mathrm{C}_{1}$ (84.2), $\mathrm{C}_{14}$ (81.4), $\mathrm{C}_{17}$ (81.2), $\mathrm{C}_{3}$ (78.5), $\mathrm{C}_{2}$ (72.2), $\mathrm{C}_{6}$ (71.4), $\mathrm{C}_{30}$ (63.2), $\mathrm{C}_{10}$ (59.3), $\mathrm{C}_{9}(56.0)$, 7-COOC (52.1), $\mathrm{C}_{29}$ (44.6), $\mathrm{C}_{4}$ (42.6), $\mathrm{C}_{5}$ (40.7), $\mathrm{C}_{13}$ (37.6), $\mathrm{C}_{15}(32.0), \mathrm{C}_{12}(26.0), \mathrm{C}_{28}(19.2), \mathrm{C}_{19}$ (17.6), $\mathrm{C}_{11}$ (16.4), and $\mathrm{C}_{18}$ (14.4).

2.4.9. Compound 9 (SM4): 6-Acetoxy-Methyl Angolensate [22]. White powder, m.p. $208-209^{\circ} \mathrm{C}$; ESI-MS m/z 529.2 $[\mathrm{M}+\mathrm{H}]^{+}\left(\mathrm{C}_{29} \mathrm{H}_{37} \mathrm{O}_{9}\right) ;{ }^{1} \mathrm{H}$ NMR (DMSO-d $\left.6,500 \mathrm{MHz}\right): \delta$ (ppm): $7.58\left(m, \mathrm{C}_{21}-\mathrm{H}\right), 7.55\left(m, \mathrm{C}_{23}-\mathrm{H}\right), 6.47\left(m, \mathrm{C}_{22}-\mathrm{H}\right)$, $5.57\left(s, \mathrm{C}_{17}-\mathrm{H}\right), 5.29\left(s, \mathrm{C}_{30}-\mathrm{H}_{\alpha}\right), 5.10\left(s, \mathrm{C}_{6,30 \beta}-\mathrm{H}\right), 3.77(s, 7-$ $\left.\mathrm{COOCH}_{3}\right), 3.67\left(d d, J=5.5,3.0 \mathrm{~Hz}, \mathrm{C}_{1}-\mathrm{H}\right), 3.02\left(s, \mathrm{C}_{5}-\mathrm{H}\right)$, $2.28\left(d d, J=14.0,3.0 \mathrm{~Hz}, \mathrm{C}_{9}-\mathrm{H}\right), 2.20\left(s, 6-\mathrm{OCOCH}_{3}\right), 1.47(s$, $\left.\mathrm{C}_{28}-\mathrm{H}\right), 1.16\left(s, \mathrm{C}_{19}-\mathrm{H}\right), 1.06\left(s, \mathrm{C}_{29}-\mathrm{H}\right)$, and $0.92\left(s, \mathrm{C}_{18}-\mathrm{H}\right)$. ${ }^{13} \mathrm{C}$ NMR (DMSO- $\left.d_{6}, 125 \mathrm{MHz}\right): \delta(\mathrm{ppm}): \mathrm{C}_{3}(211.1), \mathrm{C}_{7}$ (171.7), 6- $\mathrm{OCOCH}_{3}$ (170.4), $\mathrm{C}_{16}$ (169.6), $\mathrm{C}_{8}$ (146.9), $\mathrm{C}_{23}$ (143.9), $\mathrm{C}_{21}$ (141.7), $\mathrm{C}_{20}$ (122.3), $\mathrm{C}_{30}$ (112.4), $\mathrm{C}_{22}$ (110.8), $\mathrm{C}_{14}$ (81.7), $\mathrm{C}_{17}$ (79.9), $\mathrm{C}_{1}$ (78.9), $\mathrm{C}_{6}(73.0), 7-\mathrm{COOCH}_{3}$ (53.1), $\mathrm{C}_{9}$ (51.7), $\mathrm{C}_{4}$ (49.4), $\mathrm{C}_{5}$ (47.4), $\mathrm{C}_{10}$ (45.2), $\mathrm{C}_{13}$ (41.9), $\mathrm{C}_{2}$ (39.9), $\mathrm{C}_{15}$ (34.3), $\mathrm{C}_{12}$ (29.3), $\mathrm{C}_{28}(25.2), \mathrm{C}_{11}$ (24.8), $\mathrm{C}_{29}$ (24.4), $\mathrm{C}_{19}$ (23.0), 6- $\mathrm{OCOCH}_{3}$ (20.9), and $\mathrm{C}_{18}$ (14.3).

2.5. Antifungal Activity Bioassays. The organic soluble extracts and limonoids were tested for their in vitro antifungal activity by using the poisoned food technique. The tested materials were dissolved in DMSO or $\mathrm{MeOH}$ and then amended with a sterilized PDA medium in Petri dishes $(4 \mathrm{~cm}$ and $6 \mathrm{~cm}$ diameters) to reach to final concentrations. The solvent content was not higher than $2 \%$ in the medium, and the amended dishes were let cooling at room temperature. All of the limonoids were treated in a range concentration of $37.5-500 \mu \mathrm{g} / \mathrm{mL}$. Each Petri dish was inoculated with a mycelial plug in the center and incubated at $20-25^{\circ} \mathrm{C}$ for $2-7$ days. The Petri dishes treated with 2\% DMSO were used as negative controls. The positive controls treated with Score 250EC (a commercial fungicide containing $250 \mathrm{~g} / \mathrm{L}$ of difenoconazole, Syngenta Vietnam Co. Ltd.) were tested against $M$. oryzae, $S$. rolfsii, and oomycetes at a dose of 1000 fold dilution (equivalent to $250 \mu \mathrm{g}$ of active ingredient $/ \mathrm{mL}$ ). All experiments for each fungal species were performed at least with two replicates.

The diameter of mycelial growth was measured and recorded when fungal colonies nearly reached the edge of the negative control dishes. The percentage inhibition of mycelial growth (\%) for the tested samples was calculated by using the formula: $\%$ inhibition $=100 \times[(\mathrm{A}-\mathrm{B}) /(\mathrm{A}-4)]$ where $A$ is the diameter of the mycelial growth of fungus in the negative control dishes $(\mathrm{mm}), \mathrm{B}$ is the diameter of 
mycelial growth of fungus in treated dishes ( $\mathrm{mm})$, and 4 is the diameter of PDA plug of fungal inoculum $(\mathrm{mm})$.

The inhibition values were presented as mean$\mathrm{s} \pm$ standard deviation and evaluated by one-way analysis of variance (ANOVA). The half-maximal inhibitory concentration $\left(\mathrm{IC}_{50}, \mu \mathrm{g} / \mathrm{mL}\right)$ of limonoids against the mycelial growth of the test fungi was computed from a dose-response inhibition curve by probit analysis of WINPEPI software version 11.63.

\section{Results}

3.1. Structural Characterization of the Isolated Compounds. Compound 1 was obtained colorless crystals. The HR-ESIMS of compound 1 showed $m / z 517.2629[\mathrm{M}+\mathrm{Na}]^{+}$, (cal. for $\mathrm{C}_{30} \mathrm{H}_{38} \mathrm{O}_{6} \mathrm{Na}$ 517.2566), was established to be $\mathrm{C}_{30} \mathrm{H}_{38} \mathrm{O}_{6}$. The ${ }^{1} \mathrm{H}$ - and ${ }^{13} \mathrm{C}-\mathrm{NMR}$ spectra of compound $\mathbf{1}$ showed a $\beta$-substituted furan ring at $7.24\left(s, \mathrm{C}_{21}-\mathrm{H}\right), 7.14(d d, J=2.5$, $\left.10.5 \mathrm{~Hz}, \mathrm{C}_{23}-\mathrm{H}\right), 6.28\left(\mathrm{~s}, \mathrm{C}_{22}-\mathrm{H}\right)$, and $\mathrm{C}_{21}(142.5), \mathrm{C}_{23}(139.6)$, $\mathrm{C}_{20}$ (124.3), $\mathrm{C}_{22}$ (110.9), and $\mathrm{C}_{17}$ (51.6). The presence of two acetoxy-methyl carbons appeared at 6-OCO (170.2) and 7-OCO (170.0). Moreover, the pair of protons at 7.38 $\left(t, J=3.5 \mathrm{~Hz}, \mathrm{C}_{1}-\mathrm{H}\right)$ and $5.92\left(d d, J=3.5,10.0 \mathrm{~Hz}, \mathrm{C}_{2}-\mathrm{H}\right)$ showed two olefinic protons of double bond between $\mathrm{C}_{1}-\mathrm{C}_{2}$. The five methyl groups appeared at $0.96\left(s, \mathrm{C}_{30}-\mathrm{H}\right), 0.94$ $\left(s, \mathrm{C}_{28}-\mathrm{H}\right), 0.88\left(s, \mathrm{C}_{18^{-}} \mathrm{H}\right), 0.85\left(s, \mathrm{C}_{19^{-}} \mathrm{H}\right)$, and $0.84\left(s, \mathrm{C}_{29^{-}}\right.$ $\mathrm{H})$ in its ${ }^{1} \mathrm{H}$ NMR spectrum. To the best of our knowledge, the spectrum of compound $\mathbf{1}$ suggested the skeleton of tetracyclic triterpene (tirucallane-type triterpenoid). Thus, compound 1 (MD1) was assigned the structure of dysobinin, when its spectrums were compared to the literature $[15,16]$.

Compound 2 was obtained as white crystals. The HRESI-MS of compound 2 showed $m / z 461.3986[\mathrm{M}+\mathrm{H}]^{+}$, (cal. for $\mathrm{C}_{30} \mathrm{H}_{53} \mathrm{O}_{3} 461.3995$ ), was established to be $\mathrm{C}_{30} \mathrm{H}_{52} \mathrm{O}_{3}$. The ${ }^{1} \mathrm{H}$ NMR spectrum of compound 2 appeared the five methyl groups at $0.96\left(s, \mathrm{C}_{30}-\mathrm{H}\right), 0.94\left(s, \mathrm{C}_{28}-\mathrm{H}\right), 0.88\left(s, \mathrm{C}_{18}-\mathrm{H}\right)$, $0.85\left(s, \mathrm{C}_{19}-\mathrm{H}\right)$, and $0.84\left(s, \mathrm{C}_{29}-\mathrm{H}\right)$. However, compound 2 was oxygenated at position $3\left[3.39\left(t, J=2.5 \mathrm{~Hz}, \mathrm{C}_{3}-\mathrm{H}\right)\right.$ and $\mathrm{C}_{3}$ (76.3)]. The oxygenated methine at $\delta_{C} 71.5$ showed $\mathrm{HMBC}$ correlations to $\mathrm{C}_{26}-\mathrm{H}$ and $\mathrm{C}_{27}-\mathrm{H}$ and to the oxygenated methylene at $\delta_{C} 83.3$. The oxygenated methine at $\delta_{C}$ 86.4 showed $\mathrm{HMBC}$ correlations to $\mathrm{C}_{21}-\mathrm{H}$ and $\mathrm{C}_{17}-\mathrm{H}$. It could thus be concluded that the side chain contains an ether bridge between $\mathrm{C}_{20}$ and $\mathrm{C}_{24}$ while the second alcohol function (besides the one at $\mathrm{C}_{3}$ ) is at $\mathrm{C}_{25}$. Because $\mathrm{C}_{24}-\mathrm{H}$ shows NOEs both to $\mathrm{C}_{26}-\mathrm{H}$ and to $\mathrm{C}_{27}-\mathrm{H}$, the stereochemistry of $\mathrm{C}_{24}$ must be rel S. The NOE between $\mathrm{C}_{16}-\mathrm{H} \alpha$ and $\mathrm{C}_{23}-\mathrm{H}$ leads to the conclusion that the configuration at $\mathrm{C}_{20}$ is rel R. Again, this is in agreement with a $\mathrm{C}_{20}$-oxygenated tirucallane. The hydroxy group at $\mathrm{C}_{3}$ was determined as $\alpha$-oriented. The structure of compound 2 determined by $1 \mathrm{D}$ - and 2D-NMR experiments was in agreement as tirucallane-type triterpenoid. The database spectra of compound 2 (MD2) were identical with literature values for $(3 \alpha, 8 \mathrm{R}, 9 \mathrm{~S}, 20 \mathrm{R}, 24 \mathrm{~S})-20,24$-epoxytirucalla-3, 25diol [17].

Compound 3 was obtained as white powder. The HRESI-MS of compound 3 showed $m / z 549.2566[\mathrm{M}+\mathrm{Na}]^{+}$, (cal. for $\mathrm{C}_{30} \mathrm{H}_{38} \mathrm{O}_{8} \mathrm{Na}$ 549.2464), was established to be
$\mathrm{C}_{30} \mathrm{H}_{38} \mathrm{O}_{8}$. The NMR spectrum of compound 3 showed five tertiary methyl groups and four methyl protons. The ${ }^{13} \mathrm{C}$ NMR spectrum of compound $\mathbf{3}$ showed several similarities with that of compound $\mathbf{1}$. This indicated the presence of another tirucallane triterpene with differences in the side chain, which was evidenced only a change in the structural part of the lactone ring substitutes for the furan ring. The structure of compound 3 determined by 1D- and 2D-NMR experiments agreed as tirucallane-type triterpenoid. The spectral data of compound 3 (AP1) were identical with literature values for chisocheton compound G [18].

Compound 4 was obtained as colorless crystals. The ESIMS of compound 4 showed $m / z \quad 513.2[\mathrm{M}+\mathrm{H}]^{+}$was established to be $\mathrm{C}_{30} \mathrm{H}_{40} \mathrm{O}_{7}$. The DEPT and ${ }^{13} \mathrm{C}-\mathrm{NMR}$ spectra of compound 4 revealed 30 carbon signals, including five methylenes, seven methyl groups $(\delta 31.7,26.9,20.7,20,4,20$, $1)$, six methines, four quaternary carbons $(\delta 40.8 ; 42.9 ; 44.9$; $46.6), 4$ olefinic carbons $(\delta 119.6 ; 126.3 ; 156.8 ; 158.0)$, and four carbonyl groups $(\delta 204.4 ; 176.4 ; 170.2 ; 169.9)$. The database spectrums of compound 4 were identical with literature values for chisocheton compound E [18]. Compound 4 (AP2) was previously isolated from Chisocheton paniculatus Hiern (Meliaceae).

Compound 5 was obtained as colorless crystals. The ESIMS spectrum of compound 5 showed an ion peak at $\mathrm{m} / z$ $525.1[\mathrm{M}+\mathrm{H}]^{+}\left(\mathrm{C}_{30} \mathrm{H}_{37} \mathrm{O}_{8}\right)$. It was also clear from its $\mathrm{NMR}$ spectral data that 5 contained five methyl groups and two methyl esters. The presence of a $\beta$-furyl moiety and one methyl ester group was also identified from the spectra. The ${ }^{13} \mathrm{C}-\mathrm{NMR}$ and DEPT spectra of 5 showed signals of 29 carbons, including the peaks of 6 methyls, 5 methylenes, 8 methines, and 10 tertiary carbons. Based on these data and the comparison with the literature [19], compound 5 (AP3) is confirmed to be methyl 6-acetoxy angolensate $(6 \alpha$-acetoxyepoxyazadiradione VI).

The ESI-MS spectrum of compound 6 showed a quasimolecular ion peak at $m / z 471.1[\mathrm{M}+\mathrm{H}]^{+}\left(\mathrm{C}_{26} \mathrm{H}_{31} \mathrm{O}_{8}\right)$, which gave a molecular formula of $\mathrm{C}_{26} \mathrm{H}_{30} \mathrm{O}_{8}$. The $1 \mathrm{D}-\mathrm{NMR}$ $\left({ }^{1} \mathrm{H}-,{ }^{13} \mathrm{C}-\right)$ spectra of compound 6 revealed the presence of two double bonds and three carbonyl groups (two esters and a ketone). Moreover, 3 methyls, 6 methylenes, 8 methines, and 9 quaternary carbons were also observed. In addition, compound 6 possessed a $\beta$-furyl moiety which was characterized by its NMR spectra. Furthermore, the 2D-NMR $\left({ }^{1} \mathrm{H}-{ }^{1} \mathrm{H}\right.$ COSY and HMBC) spectra of 6 suggested that its structure was mexicanolide skeleton. The chemical shifts of $C_{1}$ (107.5) and $C_{8}(80.1)$ were confirmed the presence of the hemiacetal linkage, which has been observed in many mexicanolides. The methine proton $\mathrm{H}_{2}$ (2.84) had longrange correlations with a carbonyl group $\mathrm{C}_{3}$ (213.5), $\mathrm{C}_{1}$ (107.5), $\mathrm{C}_{30}$ (31.4), $\mathrm{C}_{10}$ (44.7), $\mathrm{C}_{4}$ (46.8), and $\mathrm{C}_{8}$ (80.1). Meanwhile, another methine proton $\mathrm{H}_{5}$ (2.32) had a cross peak with $\mathrm{C}_{6}$ (29.5), $\mathrm{C}_{28}$ (23.8), $\mathrm{C}_{29}$ (19.6), $\mathrm{C}_{4}$ (44.7), $\mathrm{C}_{3}$ (213.5), $\mathrm{C}_{7}$ (174.1), $\mathrm{C}_{10}$ (46.8), $\mathrm{C}_{19}$ (74.1), and $\mathrm{C}_{9}(61.2)$ in its $\mathrm{HMBC}$ spectrum. In addition, the studies using the spindecoupling ${ }^{1} \mathrm{H}-{ }^{1} \mathrm{H}$ COSY spectrum was identified that $\mathrm{H}_{9}$ (2.01) was coupled to $\mathrm{H}_{11 \beta}$ (1.41), $\mathrm{H}_{12 \alpha}$ (1.41), and $\mathrm{H}_{12 \beta}$ (1.76). Consequently, confirmed 6 (SM1) was found to be seneganolide [20] as shown in Figure 1. 


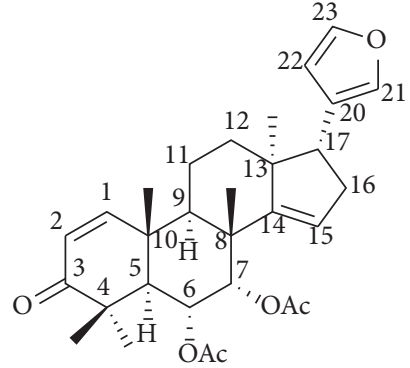

1

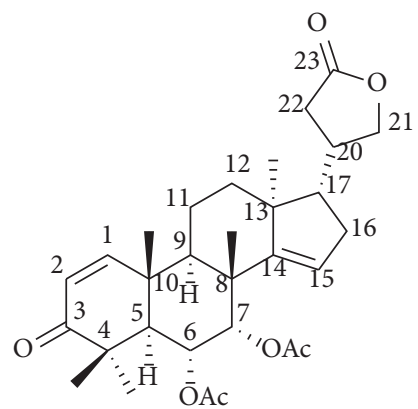

4

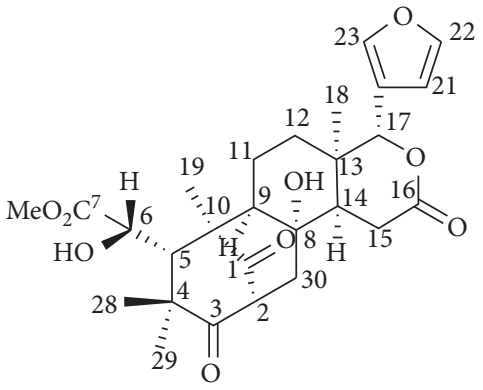

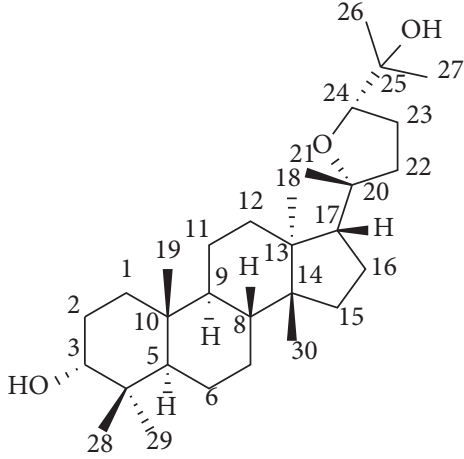

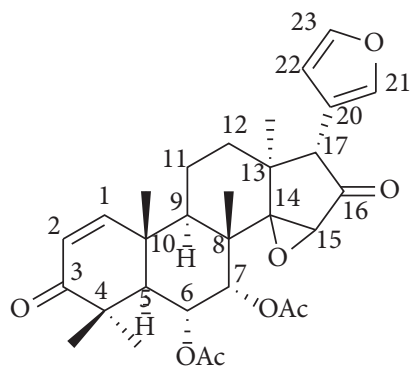

5

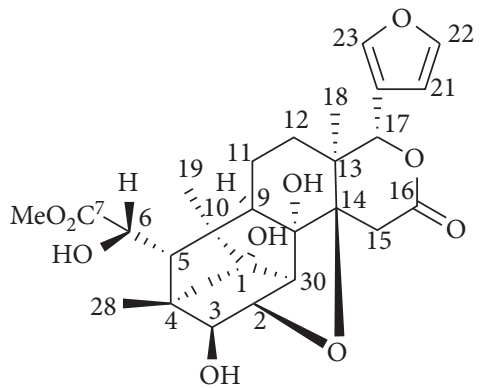

8

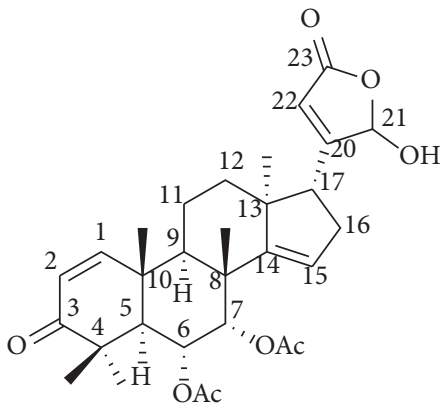

3

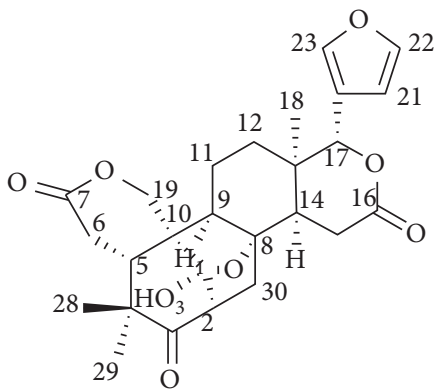

6

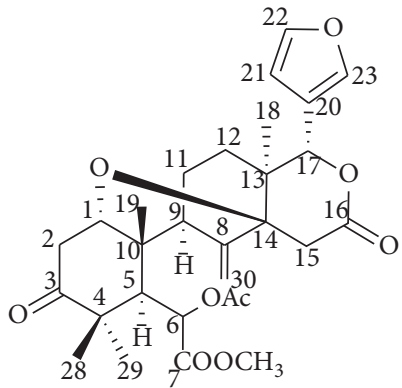

9

Figure 1: Chemical structures of the limonoids isolated from Melia dubia (1-2), Aphanamixis polystachya (3-5), and Swietenia macrophylla

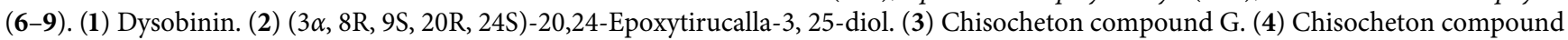
E. (5) 6 $\alpha$-Acetoxyepoxyazadiradione VI. (6) Seneganolide. (7) Khayanone. (8) Khayanolide B. (9) 6-Acetoxy-methyl angolensate.

Compound 7 was isolated as colorless crystals. Its molecular formula was established as $\mathrm{C}_{27} \mathrm{H}_{34} \mathrm{O}_{9}$ by the ESI-MS spectrum with $m / z 503.1[\mathrm{M}+\mathrm{H}]^{+}$and NMR spectral data. The structure of compound 7 could be deduced by comparing its NMR spectral data with those of compound 6 . The ${ }^{1} \mathrm{H}$ and ${ }^{13} \mathrm{C}$ NMR spectral data of 7 suggested the presence of two double bonds (furan ring) and 4 carbonyl groups ( 3 ketones and 1 ester). These NMR spectral data of compound 7 were identical with those of khayanone. Thus, we can conclude that 7 (SM2) is khayanone [21].
Compound 8 was isolated as colorless crystals. Its ESIMS indicated the molecular formula of $\mathrm{C}_{27} \mathrm{H}_{35} \mathrm{O}(\mathrm{m} / z 519.2$ $[\mathrm{M}+\mathrm{H}]^{+}$). The NMR spectra of compound $\mathbf{8}$ are mostly similar to those of compounds $\mathbf{6}$ and 7 except for some differences in the chemical shifts of $\mathrm{C}_{1}, \mathrm{C}_{19}$, and $\mathrm{C}_{3}$, suggesting 8 to have the type of phragmalin structure. The comparison of NMR data of compound $\mathbf{8}$ with the literature data [22] confirmed 8 (SM3) to be khayanolide B.

Compound 9 was purified as white powder. Its molecular formula was determined as $\mathrm{C}_{29} \mathrm{H}_{36} \mathrm{O}_{9}$ by ESI-MS. The 
structure of 9 revealed the presence of five methyl groups at $\delta_{H} 0.92 ; 1.16 ; 1.47 ; 1.06 ; 2.20$; and 3.77 by the ${ }^{1} \mathrm{H}$ NMR spectrum. Its ${ }^{13} \mathrm{C}$ NMR spectrum indicated the presence of 29 carbons, including 6 methyls, 5 methylenes, 8 methine groups, and 10 tertiary carbons. The $\beta$-furyl moiety and a methyl ester were also detected from its ${ }^{13} \mathrm{C}-\mathrm{NMR}$ and DEPT spectra. Based on these data and the comparison with literature data [22], compound 9 (SM4) is indicated to be 6acetoxy-methyl angolensate.

\subsection{In Vitro Antifungal Efficacy of the Isolated Limonoids.}

In a preliminary study, we found that the methanol extracts of M. dubia, A. polystachya, and S. macrophylla suppress the mycelial growth of various phytopathogenic fungi at 1000 and $2000 \mu \mathrm{g} / \mathrm{mL}$ (Table S1). The pure limonoids were tested for their in vitro antifungal activity against nine phytopathogenic agents including $F$. oxysporum, M. oryzae, $S$. rolfsii, $R$. solani, Alternaria spp., and $B$. cinerea, and three oomycetes Phytophthora species. The development of mycelial growth of F. oxysporum and M. oryzae was affected by almost all the test limonoids. However, F. oxysporum was less sensitive to compounds $\mathbf{1}$ and $\mathbf{5}$ on the basis of the evidence of the mycelial growth inhibition at 250 and $500 \mu \mathrm{g} /$ $\mathrm{mL}$ (Table 1). The mycelial growth of this fungus was slightly suppressed by compounds 2, 3, 4, 6, and $\mathbf{8}$ (Table 1 and Figure 2). Compound $\mathbf{3}$ gave the highest inhibition $\left(\mathrm{IC}_{50}=480.5 \mu \mathrm{g} / \mathrm{mL}\right.$ ) for F. oxysporum (Table 2). The mycelial growth of $M$. oryzae, the causal agent of rice blast, was solidity suppressed when treated with $2,3,8$, and 9 (Tables 1 and 2). At a concentration of $500 \mu \mathrm{g} / \mathrm{mL}$, compounds $\mathbf{2 , 8}$, and 9 also significantly reduced the mycelial growth of $M$. oryzae with inhibitions of $68.9 \%, 97.1 \%$, and $66.0 \%$, respectively, while compounds $\mathbf{1}, 5$, and 7 caused weak inhibitions (data not shown). The isolated limonoids 2, 3, 6, and $\mathbf{8}$ significantly suppressed the oomycetes Phytophthora spp., P. capsici, and P. palmivora at concentrations ranging from 62.5 to $500 \mu \mathrm{g} / \mathrm{mL}$ (Figure 3 and S2-4). Compounds 3 and $\mathbf{8}$ were the most potent limonoids against the tested oomycetes. The $\mathrm{IC}_{50}$ values of these four limonoids are presented in Table 2. At 250 and $500 \mu \mathrm{g} / \mathrm{mL}$, compound 8 inhibited by $69.3 \%$ and $79.7 \%$ of the mycelial growth of Phytophthora spp., respectively (data not shown). Compound 3 displayed a dose-dependent activity against the test oomycetes Phytophthora spp. and P. capsici (Figure 2). This compound was also most active against Phytophthora spp and P. capsici; it caused $75.71 \%$ and $70.66 \%$ inhibition for the mycelial growth of the test oomycetes at 250 and $500 \mu \mathrm{g} / \mathrm{mL}$, respectively (Figure S2 and 3). Similar to the antifungal activity of $\mathbf{3}$, compound $\mathbf{8}$ also had a dose-dependent activity against Phytophthora spp. and P. capsici. However, it seems that the fungal efficacy of $\mathbf{8}\left(\mathrm{IC}_{50}=97.1 \mu \mathrm{g} / \mathrm{mL}\right)$ was better than that of $3\left(\mathrm{IC}_{50}=178.5 \mu \mathrm{g} / \mathrm{mL}\right)$ when tested against Phytophthora spp. In contrast, the plant pathogens F. oxysporum, $M$. oryzae, and $P$. palmivora were strongly inhibited in the treatment with 3 compared with those with 8 (Figure 2, S2-4, and Table 2).

Besides, $S$. rolfsii was strongly inhibited by limonoids 2 , 3, 6, and $\mathbf{8}$ in vitro (Table 1 and Figure 2). All of the four
TABle 1: Control value (\%) of limonoids 1, 4, 5, 7, and 9 against Fusarium oxysporum, Magnaporthe oryzae, and Sclerotium rolfsii.

\begin{tabular}{lcccccc}
\hline \multirow{2}{*}{ Fungi } & $\begin{array}{c}\text { Conc. } \\
(\mu \mathrm{g} / \mathrm{mL})\end{array}$ & $\mathbf{1}^{\mathrm{b}}$ & $\mathbf{4}$ & $\mathbf{5}$ & $\mathbf{7}$ & $\mathbf{9}$ \\
\hline \multirow{2}{*}{$\mathrm{FO}^{\mathrm{a}}$} & 250 & $11.73 \pm 7.64^{\mathrm{c}}$ & $28.67 \pm 2.38$ & $18.51^{\mathrm{d}}$ & $\mathrm{nt}$ & $\mathrm{nt}$ \\
& 500 & $25.03 \pm 5.93$ & $30.63 \pm 0.58$ & 22.43 & $\mathrm{nt}$ & $\mathrm{nt}$ \\
$\mathrm{MG}$ & 250 & $13.84 \pm 5.97$ & $24.89 \pm 0.69$ & 25.67 & 6.60 & 14.15 \\
& 500 & $27.67 \pm 3.14$ & $33.33 \pm 0.42$ & 28.61 & 16.04 & 66.04 \\
$\mathrm{SR}$ & 250 & $\mathrm{Nt}$ & $\mathrm{nt}$ & $\mathrm{nt}$ & $\mathrm{nt}$ & $\mathrm{nt}$ \\
& 500 & $\mathrm{Nt}$ & $\mathrm{nt}$ & $\mathrm{nt}$ & $\mathrm{nt}$ & $\mathrm{nt}$ \\
\hline
\end{tabular}

${ }^{\mathrm{a}} \mathrm{FO}:$ Fusarium oxysporum, MG:Magnaporthe oryzae, and SR:Sclerotium rolfsii. ${ }^{\mathrm{b}}$ Compounds: $\mathbf{1}$, dysobinin; $\mathbf{4}$, chisocheton compound E; 5, $6 \alpha-$ acetoxyepoxyazadiradione VI; 7, khayanone; 9, 6-acetoxy-methyl angolensate. ${ }^{\circ}$ The values are the average of two determinations \pm standard error of the mean. nt: not tested. ${ }^{\mathrm{d}}$ Values are obtained through a single treatment.

compounds displayed a dose-dependent antifungal activity against $S$. rolfsii. Compounds $\mathbf{2}$ and $\mathbf{6}$ were the best inhibitor against the mycelial growth of $S$. rolfsii with $\mathrm{IC}_{50}$ values of 94.0 and $79.4 \mu \mathrm{g} / \mathrm{mL}$, respectively. Compounds 3 and 8 also strongly inhibited this fungus with $\mathrm{IC}_{50}$ values of 128.0 and $124.5 \mu \mathrm{g} / \mathrm{mL}$, respectively (Table 2 ).

Of the limonoids isolated from S. macrophylla (6-9), compounds 6-8 belong to mexicanolide-type limonoids. Compound $\mathbf{6}$ moderately inhibited the mycelial growth of $R$. solani (57.9\%) while 8 did not when tested at a concentration of $250 \mu \mathrm{g} / \mathrm{mL}$ (data not shown). Compounds 6 (seneganolide) and 8 (khayanolide B) affected the mycelial growth of various oomycetes and fungi (Table 2 and Figure 2). Compounds 7 and 9 were isolated in a limited amount and were tested only with $M$. oryzae. Of those, limonoid 9 belonging to andirobin-type limonoid was highly active against $M$. oryzae.

Compounds $\mathbf{1}$ and $\mathbf{2}$ were isolated from M. dubia in the work, and their antifungal activity was evaluated against various phytopathogenic fungi. Dysobinin (1) was tested against only $F$. oxysporum and $M$. oryzae because of lacking isolated weight. At a concentration of $500 \mu \mathrm{g} / \mathrm{mL}$, compound 2 exhibited discernible effects on F. oxysporum (32.4\%), $M$. oryzae (68.8\%), Phytophthora spp. (46.4\%), and P. capsici (30.2\%) (data not shown). Compared with 2, compound dysobinin (1) was less active against $F$. oxysporum and $M$. oryzae. The mycelial growth of $B$. cinerea, $R$. solani, and Alternaria spp. was also weakly inhibited by compounds 2, 3, 6 , and 8 with an inhibition range of $12.8-34.8 \%$ at concentrations of 250 and $500 \mu \mathrm{g} / \mathrm{mL}$ (data not shown). All positive controls against the mycelial growth of $M$. oryzae, $S$. rolfsii, and oomycetes exhibited by $100 \%$ suppression when treated with difenoconazole at $250 \mu \mathrm{g} / \mathrm{mL}$ (data not shown).

\section{Discussion}

Limonoids are known as the main secondary metabolites produced by the plants from Melia and Citrus genera. The insecticidal limonoids have been applied to pest management in agriculture for a long time. However, the reports of the antifungal activity of limonoids from Meliaceae family have been rare until now. The notable antifungal activity of the three methanol extracts of M. dubia, A. polystachya, and 


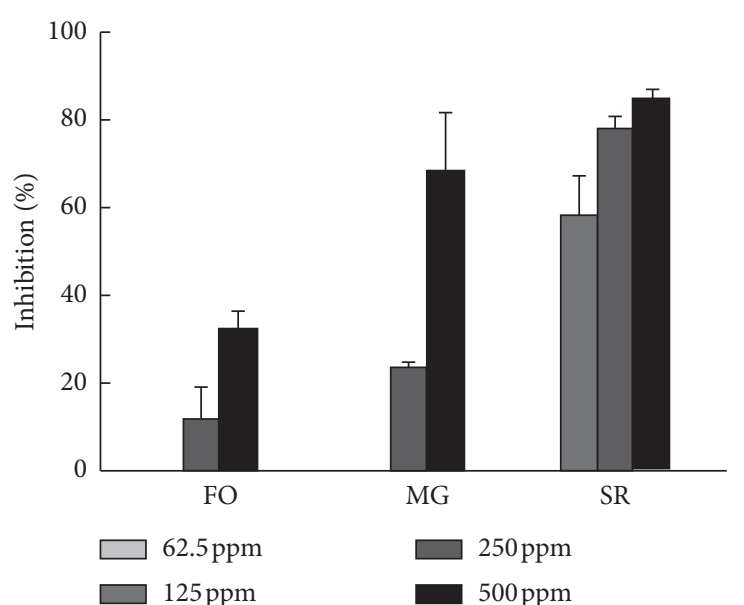

(a)

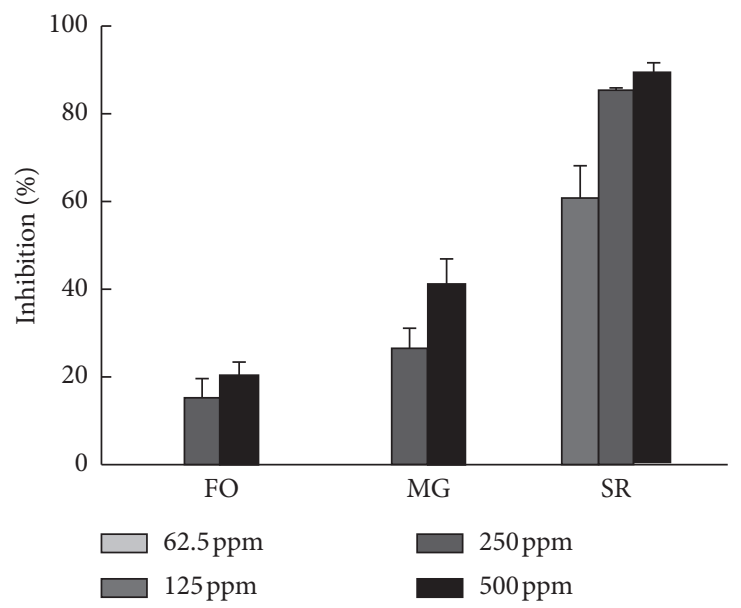

(c)

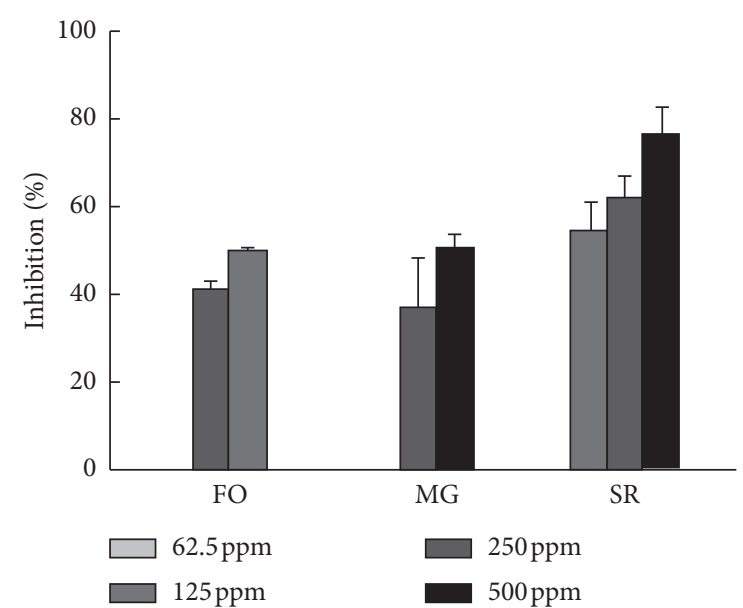

(b)

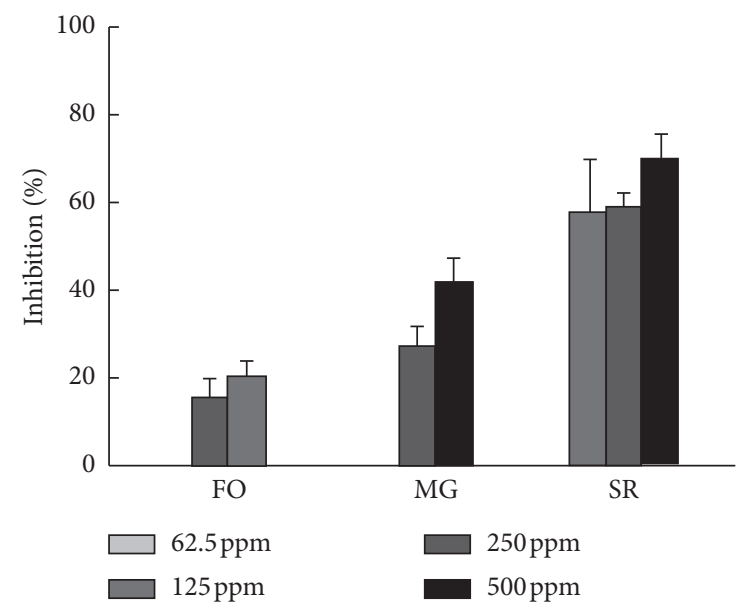

(d)

FIGURE 2: In vitro antifungal activity of the isolated limonoids $\mathbf{2 , 3}, \mathbf{6}$, and $\mathbf{8}$ against three phytopathogenic fungi. FO: Fusarium oxysporum; MG : Magnaporthe oryzae; SR: Sclerotium rolfsii. (a) Compound 2. (b) Compound 3. (c) Compound 6. (d) Compound 8.

S. macrophylla has prompted us to search the antifungal ingredients in the samples and evaluate their in vitro antifungal efficacy against phytopathogenic fungi. In our study, nine limonoids were tested for their antifungal activity against nine phytopathogenic fungi. Five potent antifungal limonoids 2, 3, 6, 8, and 9 significantly inhibited the test fungi in a broad spectrum. S. rolfsii was also found to be the best sensitive to the isolated limonoids $\mathbf{2 , 3}, \mathbf{6}$, and $\mathbf{8}$; for this fungus, all of the compounds displayed a strong antifungal activity in a dose-dependent manner and $\mathrm{IC}_{50}$ values in a range of 79.4-128.0 $\mu \mathrm{g} / \mathrm{mL}$ (Figure 2 and Table 2). F. oxysporum seemed to be less sensitive to all test fungi, except for compound 3 when tested at a concentration of $500 \mu \mathrm{g} / \mathrm{mL}$. Remarkably, compounds $\mathbf{3}$ and $\mathbf{8}$ were the most active and showed a broad-spectrum antifungal activity against various fungal plant pathogens such as F. oxysporum, M. oryzae, and $S$. rolfsii, and the three oomycete strains of Phytophthora species. Compounds 1-5 belong to the class of ring D-opened limonoid, and the class includes some antifungal limonoids such as 3-deacetylkhivorin, 1, 3, 7-trideacetylkhivorin, 7-deacetylgedunin, and 7-deacetoxy-7- oxogedunin isolated from Khaya ivorensis and reported to be weakly and moderately active against the plant fungus Botrytis cinerea at the concentrations higher than $1000 \mu \mathrm{g} /$ $\mathrm{mL}[10]$.

A. polystachya grows abundantly in India, China, and Southeast Asia and has been used as medicinal materials in traditional medicine. The structural characterization and bioactivities of various limonoids from the fruits of $A$. polystachya were investigated in previous studies $[3,23,24]$. Especially, the reports of the antifungal activity of limonoids from this plant have been rare until now. Srivastava et al. (2003) reported the isolation of an andirobin-type limonoid named amoorinin-3-O- $\alpha$-L-rhamnopyranosyl-(1-6)- $\beta$-Dglucopyranoside from $A$. polystachya with antifungal activity against Aspergillus niger and Candida albicans [25]. In a previous study by Zhang et al. (2013), A, B-seco limonoids, aphapolynin $\mathrm{C}$, rohituka-15, aphanamolide $\mathrm{A}$, and aphapolynin A from the fruits of A. polystachya were evaluated antifungal property against various phytopathogenic fungi such as Alternaria solani, Botryotinia fuckeliana, Gibberella 
TABLE 2: The half-maximal inhibitory concentration $\left(\mathrm{IC}_{50}, \mu \mathrm{g} / \mathrm{mL}\right)$ of limonoids $\mathbf{2}, \mathbf{3}, \mathbf{6}, \mathbf{8}$, and 9 against the mycelial growth of phytopathogenic fungi and oomycetes in vitro.

\begin{tabular}{lccccc}
\hline \multirow{2}{*}{ Fungi } & \multicolumn{4}{c}{ The half-maximal inhibitory concentration $\left(\mathrm{IC}_{50}\right)(\mu \mathrm{g} / \mathrm{mL})$} \\
& $\mathbf{2}$ & $\mathbf{3}$ & $\mathbf{6}$ & $\mathbf{8}$ & $\mathbf{9}$ \\
\hline FO & $>500$ & $480.5 \pm 20.9$ & $>500$ & $\mathrm{nt}$ \\
MG & $386.3 \pm 2.8$ & $490.0 \pm 21.1$ & $>500$ & $318.3 \pm 1.2$ & $422.8 \pm 3.9$ \\
SR & $94.0 \pm 2.5$ & $128.0 \pm 3.6$ & $79.4 \pm 2.1$ & $124.5 \pm 4.9$ & $\mathrm{nt}$ \\
PS & $>500$ & $178.5 \pm 4.2$ & $434.3 \pm 10.8$ & $97.1 \pm 3.4$ & $\mathrm{nt}$ \\
PC & $>500$ & $262.4 \pm 10.6$ & $>500$ & $280 \pm 11.4$ & $\mathrm{nt}$ \\
PP & $\mathrm{nt}$ & $351.5 \pm 9.6$ & $>500$ & $\mathrm{nt}$ \\
\hline
\end{tabular}

${ }^{\mathrm{a}} \mathrm{FO}$ : Fusarium oxysporum; MG:Magnaporthe oryzae; SR:Sclerotium rolfsii BV; PS:Phytophthora spp.; PC:Phytophthora capsici; PP: Phytophthora pal-

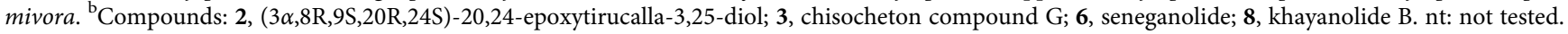

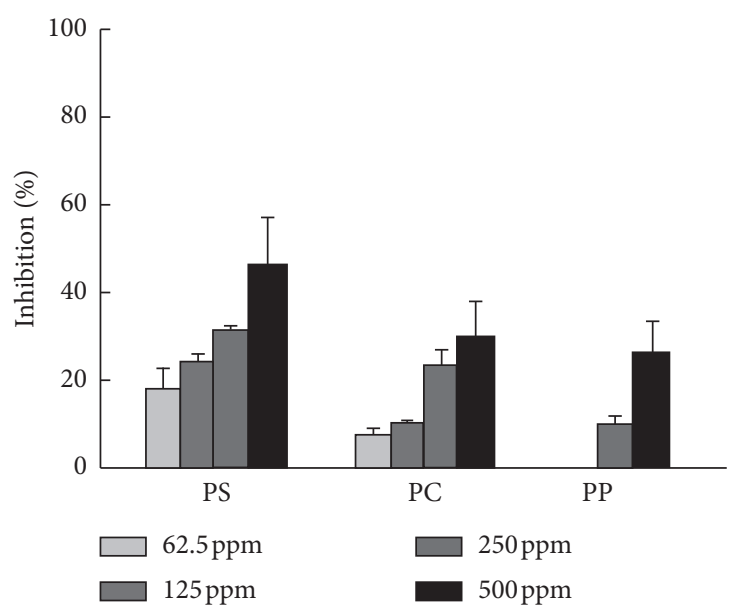

(a)

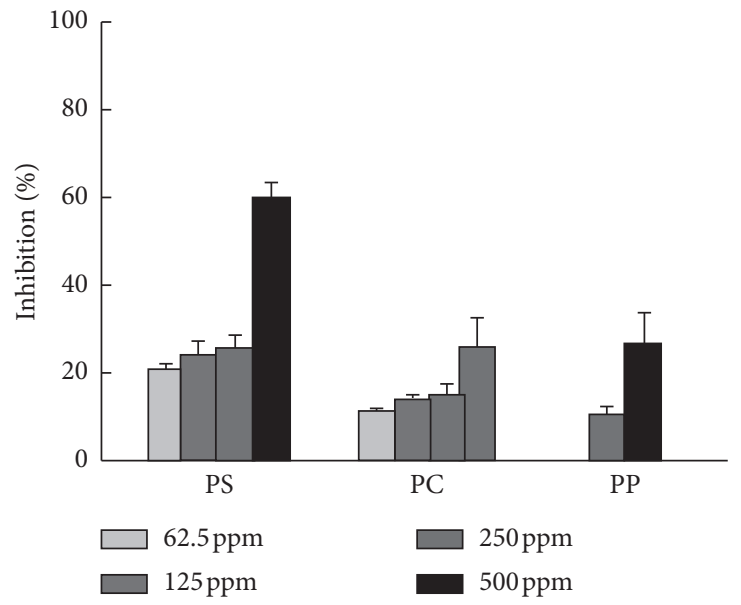

(c)

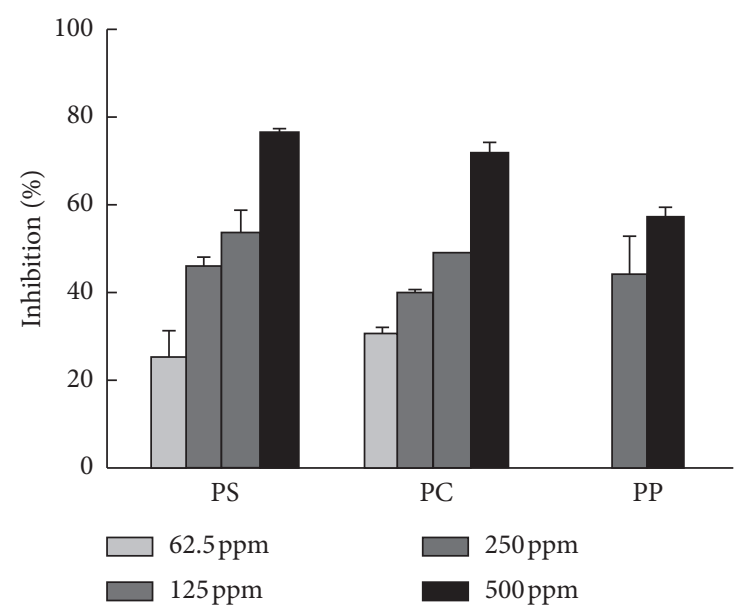

(b)

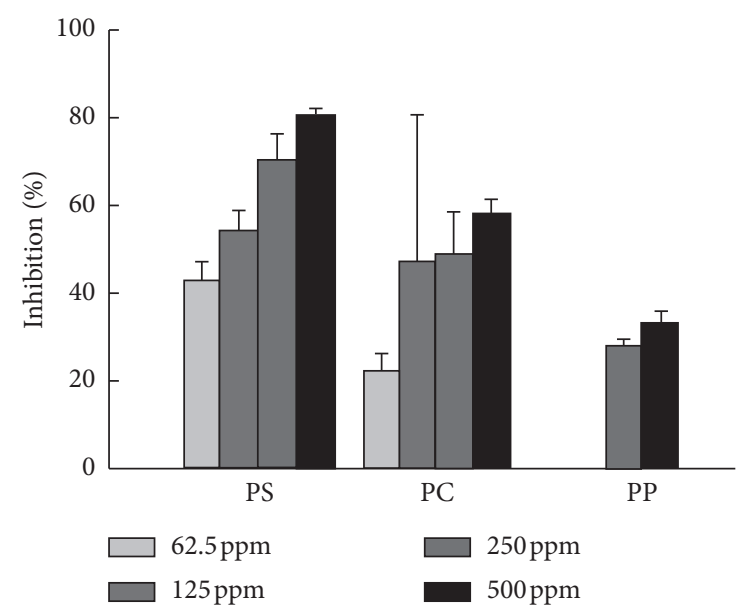

(d)

FIgUre 3: In vitro antifungal activity of the isolated limonoids 2, 3, 6, and $\mathbf{8}$ against oomycete Phytophthora strains. PS: Phytophthora spp.; PC: Phytophthora capsici; PP : Phytophthora palmivora. (a) Compound 2. (b) Compound 3. (c) Compound 6. (d) Compound 8.

zeae, Septoria tritici, P. infestans, Pythium dissimile, and Uromyces viciae-fabae [14]. Compounds rohituka-15 and aphanamolide A completely inhibited $P$. dissimile, and aphapolynin A did partial inhibition at a concentration of $20 \mu \mathrm{g} / \mathrm{mL}$. Aphapolynin C and aphapolynin A displayed strong inhibition against $U$. viciae-fabae at $100 \mu \mathrm{g} / \mathrm{mL}$. However, all of the test compounds were inactive against oomycete $P$. infestans at concentrations of 200 and $60 \mu \mathrm{g} / \mathrm{mL}$.
In contrast, we reported here that compounds $\mathbf{3 , 4}$, and $\mathbf{5}$ are isolated from $A$. polystachya and have a similar carbon skeleton except for the substitute attached to C-17 (4hydroxybut-2-enolide for 3 , butanolide for $\mathbf{4}$, and furan ring for 5). Chisocheton compound G (3) showed the best mycelial growth inhibition against Phytophthora species, while $\mathbf{4}$ and $\mathbf{5}$ were almost inactive against the oomycete. This may be due to the structure of $\mathbf{3}$ contains 4-hydroxybut-2-enolide 
moiety at C-17, which is similar to the head moiety of annonaceous acetogenins with a potential of antioomycete activity and cytotoxicity [26].

Swietenia species biosynthesize more than 160 limonoids including 77 mexicanolide-type limonoids (most from $S$. mahagoni and S. macrophylla). The Meliaceous plant $S$. macrophylla grows widely in Central and South American countries, India, Malaysia, and China $[1,4]$. It is rare to see in Vietnam, and the phytochemical investigation, as well as antifungal properties of the local species, has not been reported much. From the dried fruits of S. macrophylla growing in Vietnam, four limonoids 6-9 were isolated by various chromatographic methods. Out of them, mexicanolide-type limonoids seneganolide (6) and khayanolide B (8) showed the best antioomycete activity against Phytophthora species. Especially, khayanolide B (8) was the most active against $M$. oryzae, Phytophthora spp., and $P$. capsici. In the same group of mexicanolide carbon skeleton, three limonoids seneganolide A, 2-acetoxyseneganolide A, and methyl 6-hydroxyangolensate were reported to isolate from the fruits of Khaya senegalensis and tested against phytopathogenic fungus Botrytis cinerea at relatively high concentrations of 1000 and $1500 \mu \mathrm{g} / \mathrm{mL}$. Limonoid 2acetoxyseneganolide A suppressed the mycelial growth of $B$. cinerea at $1000 \mu \mathrm{g} / \mathrm{mL}(61.50 \%)$ and $1500 \mu \mathrm{g} / \mathrm{mL}(68.33 \%)$, while seneganolide A and methyl 6-hydroxyangolensate showed a weaker inhibition [9]. Khayanolide B (8) was also reported as an antifeedant substance against Spodoptera littoralis with an $\mathrm{EC}_{50}$ of $2.19 \mathrm{mg} / \mathrm{kg}$ [2]. Limonoid swietenolide isolated from S. macrophylla was documented to have antifeedant activity with antifeedant index of 94.1 against fall armyworm (Spodoptera frugiperda). 6-O-acetylswietenolide, 3, 6-O, O-diacetylswietenolide, and swietemahonin $\mathrm{F}$ exhibited antifeedant indices of $72.2,72.0$, and 70.2, respectively [1]. According to Sun et al. (2018), swietenine from this plant also showed the greatest potency with a $50 \%$ antifeedant index concentration of 2.49 against $S$. frugiperda [4].

As for the source rich in limonoids in Meliaceae species, A. indica and M. dubia have been described as potential plant systems that contain a wide range of bioactive limonoids that are both chemically and structurally complex [27]. Salannin, a well-known insecticidal limonoid, was found to be produced by A. indica and M. dubia. The bark of M. dubia was also reported to contain cytotoxic euphane-type triterpenes against P388 cancer cells [7]. However, there are no reports on antifungal activity of $M$. dubia against phytopathogenic fungi up to now. In a preliminary study by Mahmoud et al. (2011), the extracts derived from the leaves and seeds of $A$. indica were assessed for antifungal activity against six human fungal pathogens Aspergillus flavus, $A$. fumigatus, A. Niger, A. terreus, C. albicans, and M. gypseum [28]. Nimonol with a molecular formula of $\mathrm{C}_{28} \mathrm{H}_{36} \mathrm{O}_{5}$ was separated and identified as the main compound of ethyl acetate extract of $A$. indica, but it displayed no inhibitory effect on all the six fungal pathogens tested [28]. In contrast, Alvarez-Caballero and Coy-Barrera (2019) described that azadirone-type limonoids such as nimonol, 14, 15-epoxynimonol, isomeldenin, zafaral, and O-acetyl-7- deacetylnimocinol isolated from $A$. indica possess a good inhibition against conidia germination of $F$. oxysporum. Nimonol was the best inhibitor; of which inhibition against F. oxysporum conidia germination was observed at $\mathrm{IC}_{50}$ of $1.48 \mu \mathrm{M}$. Alvarez-Caballero and Coy-Barrera (2019) also demonstrated that the presence of a 14, 15-epoxy group and the saturation at 1,2-olefilic positions in the structure of azadirone-type limonoids led to a diminished effect on fungal conidia germination [11]. In our study, 1, 3, 4, and 5 were isolated and identified as azadirone-type limonoids. Among them, compound 3 showed a broad-spectrum activity with potent suppression against almost all of the test fungi in the concentration ranging from 37.5 to $500 \mu \mathrm{g} / \mathrm{mL}$ (Table 2 and Figure 2), while compounds 1, 4 and 5 showed a little effect on the growth of F. oxysporum and M. grisea. This suggested an implication of structure-antifungal activity relationship of the studied azadirone-type limonoids. The 2hydroxybutanolide groups at C-17 in the structure of 3 may make an enhancing effect on inhibition against fungi. Concerned to the antifungal activity of azadirone-type limonoids, $6 \alpha$-acetoxyazadirone and 1, 2-dihydro- $6 \alpha$-acetoxyazadirone were also briefly reported to possess strong antifungal activity against pathogenic fungi Curvularia verruciformis, Dreschleva oryzae, and A. solani, but no information of inhibition effectiveness, biological testing methods, and test concentration was provided in the article $[8,29]$. According to Govindachari et al. (1998), the neem oil derived from the seeds of $A$. indica showed antifungal activity against phytopathogenic fungi and contains an abundance of limonoids such as azadiradione, nimbin, 6deacetylnimbin, salannin, and epoxyazadiradione [12]. However, these compounds in pure form have not much affected the mycelial growth of $D$. oryzae, F. oxysporum f.sp. vasinfectum, and Alternaria tenuis at a high concentration of $1000 \mu \mathrm{g} / \mathrm{mL}$. Only 6-deacetylnimbin and nimbin inhibited D. oryzae, the causal agent of rice brown leaf spot disease, by 63.3 and $64.8 \%$, respectively. Besides, either additive or synergistic influence of the mixture containing those terpenoids was observed with an inhibition of $70 \%$ for $D$. oryzae at $1000 \mu \mathrm{g} / \mathrm{mL}$ [12].

As for the antifungal effects on soilborne plant pathogenic fungi, Sharma et al. (2003) reported that azadirachtins $\mathrm{A}, \mathrm{B}$, and $\mathrm{H}$ possessing antifungal potential against $R$. solani and $S$. rolfsii were efficiently isolated from the seed kernels of $A$. indica. Azadirachtin A inhibited the mycelial growth of $R$. solani and S. rolfsii at $\mathrm{ED}_{50}$ values of $104.8 \mu \mathrm{g} / \mathrm{mL}$ (purity of $90 \%$ for azadirachtin A) and $93.6 \mu \mathrm{g} / \mathrm{mL}$, respectively. Azadirachtins $\mathrm{B}$ and $\mathrm{H}$ exhibited a better inhibition with $\mathrm{ED}_{50}$ values varying from 43.9 to $85.9 \mu \mathrm{g} / \mathrm{mL}$ for the two fungi [30]. Therefore, in addition to azadirachtins A, B, and $\mathrm{H}$ belonging to the ring $\mathrm{C}$-seco limonoid group, the isolated compounds $2,3,6$, and 8 in our study are different structure type limonoids with potent inhibition against $S$. rolfsii were consequently reported.

Our study has described the antifungal property of the limonoids isolated from A. polystachya, M. dubia, and S. macrophylla against various phytopathogenic fungi for the first time. To discover more potent antifungal limonoids, experiments in the mode of action and the relationship 
between structures and antifungal activity of limonoids need to conduct. In further studies, the in vivo antifungal efficacy and phytotoxicity of the bioactive limonoids, which could be formulated evenly into a ready-to-use formulation, should be evaluated to determine their potential disease control efficacy on various crops.

\section{Conclusions}

Considering the application of limonoids in crop protection, in our work, the chemical structural characterization and antifungal activity evaluation of nine limonoids isolated from M. dubia (1-2), A. polystachya (3-5), and S. macrophylla (6-9) were described and pointed our promising active compounds against various phytopathogenic fungi. Limonoids 2, 3, 6, 8, and 9 were found to be promising candidates with a broad antifungal spectrum. Compounds $\mathbf{2}$, $\mathbf{8}$, and $\mathbf{9}$ displayed moderate activity against $M$. oryzae, while S. rolfsii and Phytophthora species were the most susceptible species to the test limonoids. It is noteworthy that chisocheton compound G (3) isolated from A. polystachya and khayanolide B (8) isolated from S. macrophylla were the most potent antifungal limonoids against $M$. oryzae and Phytophthora species. Our findings may suggest and encourage more work on the discovery of antifungal limonoids and lead compounds from the group of limonoids.

\section{Data Availability}

The data used to support the findings of this study are included in the supplementary materials and available from the corresponding authors upon request.

\section{Conflicts of Interest}

The authors declare no conflicts of interest.

\section{Authors' Contributions}

Thang Tran Dinh, Hoang Dinh Vu, Dang Ngoc Quang, and Quang Le Dang were responsible for conceptualization, methodology, and supervision. Thanh Nguyen Tan, Hieu Tran Trung, Tuan Nguyen Ngoc, Hien Vu Thi, Dang Ngoc Quang, and Thang Tran Dinh were responsible for extraction, purification, and structure identification for isolated compounds. Thanh Huong Nguyen, Hoai Thu Thi Do, and Quang Le Dang were responsible for antifungal bioassay and biological data analysis. Thanh Nguyen Tan, Hoai Thu Thi Do, Quang Le Dang, and Thang Tran Dinh were responsible for writing - original draft preparation, and editing. All authors read and approved the submitted manuscript.

\section{Acknowledgments}

The authors thank Thanh Huong Nguyen for the bioassay's assistance. This research was funded by the Vietnam $\mathrm{Na}$ tional Foundation for Science and Technology Development (NAFOSTED) under grant no. 104.01-2018.315.

\section{Supplementary Materials}

Figure S1a: isolation scheme of isolated compounds 1-2 from the fruits of Melia dubia. Figure S1b: isolation scheme of isolated compounds 3-5 from the fruits of Aphanamixis polystachya. Figure S1c: isolation scheme of isolated compounds 6-9 from the fruits of Swietenia macrophylla. Figure S2: in vitro antifungal activity of the most active compounds $\mathbf{3}$ and $\mathbf{8}$ against the mycelial growth of Phytophthora spp. Figure S3: in vitro antifungal activity of the most active compounds $\mathbf{3}$ and $\mathbf{8}$ against the mycelial growth of Phytophthora capsici. Figure S4: in vitro antifungal activity of the most active compounds 3, 6, and 8 against the mycelial growth of Phytophthora palmivora. Figure S5: in vitro antifungal activity of compound $\mathbf{2}$ against the mycelial growth of Magnaporthe oryzae. Figure S6: in vitro antifungal activity of the most active compounds 2, 3, 6, and 8 against the mycelial growth of Sclerotium rolfsii. Table S1: in vitro inhibitory activity of $\mathrm{MeOH}$ extracts from Melia dubia, Aphanamixis polystachya, and Swietenia macrophylla against Magnaporthe oryzae, Phytophthora capsici, and Sclerotium rolfsii. (Supplementary Materials)

\section{References}

[1] S. Moghadamtousi, B. Goh, C. Chan, T. Shabab, and H. Kadir, "Biological activities and phytochemicals of Swietenia macrophylla king," Molecules, vol. 18, no. 9, pp. 10465-10483, 2013.

[2] Q.-G. Tan and X.-D. Luo, "Meliaceous limonoids: chemistry and biological activities," Chemical Reviews, vol. 111, no. 11, pp. 7437-7522, 2011.

[3] A. Roy and S. Saraf, "Limonoids: overview of significant bioactive triterpenes distributed in plants kingdom," Biological and Pharmaceutical Bulletin, vol. 29, no. 2, pp. 191-201, 2006.

[4] Y. P. Sun, W. F. Jin, Y. Y. Wang et al., "Chemical structures and biological activities of limonoids from the genus Swietenia (Meliaceae)," Molecules (Basel, Switzerland), vol. 23, 2018.

[5] A. J. Mordue and A. J. Nisbet, "Azadirachtin from the neem tree Azadirachta indica: its action against insects," Anais da Sociedade Entomológica do Brasil, vol. 29, pp. 615-632, 2000.

[6] Q. L. Dang, C.-H. Lim, and J.-C. Kim, "Current status of botanical pesticides for crop protection," Research in Plant Disease, vol. 18, no. 3, pp. 175-185, 2012.

[7] L. Zhao, C.-H. Huo, L.-R. Shen, Y. Yang, Q. Zhang, and Q.-W. Shi, "Chemical constituents of plants from the GenusMelia," Chemistry and Biodiversity, vol. 7, no. 4, pp. 839-859, 2010.

[8] W. Zhao, J.-L. Wolfender, K. Hostettmann, R. Xu, and G. Qin, "Antifungal alkaloids and limonoid derivatives from Dictamnus dasycarpus,” Phytochemistry, vol. 47, no. 1, pp. 7-11, 1998.

[9] S. A. M. Abdelgaleil, T. Iwagawa, M. Doe, and M. Nakatani, "Antifungal limonoids from the fruits of Khaya senegalensis," Fitoterapia, vol. 75, no. 6, pp. 566-572, 2004.

[10] S. A. Abdelgaleil, F. Hashinaga, and M. Nakatani, "Antifungal activity of limonoids fromKhaya ivorensis," Pest Management Science, vol. 61, no. 2, pp. 186-190, 2005.

[11] J. M. Álvarez-Caballero and E. Coy-Barrera, "Chemical and antifungal variability of several accessions of Azadirachta 
indica A. Juss. from six locations across the Colombian Caribbean coast: identification of antifungal azadirone limonoids," Plants, vol. 8, p. 555, 2019.

[12] T. R. Govindachari, G. Suresh, G. Gopalakrishnan, B. Banumathy, and S. Masilamani, "Identification of antifungal compounds from the seed oil ofAzadirachta Indica," Phytoparasitica, vol. 26, no. 2, pp. 109-116, 1998.

[13] T. R. Govindachari, G. Suresh, B. Banumathy, S. Masilamani, G. Gopalakrishnan, and G. N. Krishna Kumari, "Antifungal activity of some B,D-seco limonoids from two Meliaceous plants," Journal of Chemical Ecology, vol. 25, no. 4, pp. 923-933, 1999.

[14] Y. Zhang, J.-S. Wang, X.-B. Wang et al., "Limonoids from the fruits of Aphanamixis polystachya (Meliaceae) and their biological activities," Journal of Agricultural and Food Chemistry, vol. 61, no. 9, pp. 2171-2182, 2013.

[15] S. Laphookhieo, W. Maneerat, S. Koysomboon, R. Kiattansakul, K. Chantrapromma, and J. K. Syers, "A novel limonoid from the seeds of Chisocheton siamensis," Canadian Journal of Chemistry, vol. 86, no. 3, pp. 205-208, 2008.

[16] S. Singh, H. S. Garg, and N. M. Khanna, "Dysobinin, a new tetranortriterpene from Dysoxylum binectariferum," Phytochemistry, vol. 15, no. 12, pp. 2001-2002, 1976.

[17] L. G. Hippolyt, K. Marcel, B. Reto, and J. S. Thomas, “Terpenoids from the Oleo-Gum-Resin of Boswellia serrata and their antiplasmodial effects in vitro," Planta Medica, vol. 83, pp. 1214-1226, 2017.

[18] J. D. Connolly, C. Labbé, D. S. Rycroft, and D. A. H. Taylor, "Tetranortriterpenoids and related compounds. Part 22. New apotirucailol derivatives and tetranortriterpenoids from the wood and seeds of chisocheton paniculatus(meliaceae)," Journal of the Chemical Society, Perkin Transactions, vol. 1, pp. 2959-2964, 1979.

[19] D. Lavie, E. C. Levy, and R. Zelnik, "The constituents of Carapa guianensis Aubl. and their biogenetic relationship," Bioorganic Chemistry, vol. 2, no. 1, pp. 59-64, 1972.

[20] M. Nakatani, S. A. M. Abdelgaleil, H. Okamura, T. Iwagawa, and M. Doe, "Seneganolide, a novel antifeeding mexicanolide fromKhaya senegalensis," Chemistry Letters, vol. 29, no. 8, pp. 876-877, 2000.

[21] H. Zhang, J. Tan, D. Vanderveer, X. Wang, M. J. Wargovich, and F. Chen, "Khayanolides from african mahogany Khaya senegalensis (meliaceae): a revision," Phytochemistry, vol. 70, no. 2, pp. 294-299, 2009.

[22] S. A. M. Abdelgaleil, H. Okamura, T. Iwagawa et al., "Khayanolides, rearranged phragmalin limonoid antifeedants from Khaya senegalensis," Tetrahedron, vol. 57, no. 1, pp. 119-126, 2001.

[23] J.-Y. Cai, D.-Z. Chen, S.-H. Luo et al., "Limonoids from Aphanamixis polystachya and their antifeedant activity," Journal of Natural Products, vol. 77, no. 3, pp. 472-482, 2014.

[24] C. M. Camero, A. Vassallo, M. De Leo, A. Temraz, N. De Tommasi, and A. Braca, "Limonoids from Aphanamixis polystachya leaves and their interaction with Hsp90," Planta Medica, vol. 84, no. 12/13, pp. 964-970, 2018.

[25] S. K. Srivastava, S. D. Srivastava, and S. Srivastava, "New biologically active limonoids and flavonoids from Aphanamixis polystachya," Indian Journal of Chemistry -Section B, vol. 42B, no. 12, pp. 3155-3158, 2003.

[26] Q. L. Dang, W. K. Kim, C. M. Nguyen et al., "Nematicidal and antifungal activities of annonaceous acetogenins fromAnnona squamosaagainst various plant pathogens," Journal of Agricultural and Food Chemistry, vol. 59, no. 20, pp. 11160-11167, 2011.
[27] V. Paritala, K. K. Chiruvella, C. Thammineni, R. G. Ghanta, and A. Mohammed, "Phytochemicals and antimicrobial potentials of mahogany family," Revista Brasileira de Farmacognosia, vol. 25, no. 1, pp. 61-83, 2015.

[28] D. A. Mahmoud, N. M. Hassanein, K. A. Youssef, and M. A. Abou Zeid, "Antifungal activity of different neem leaf extracts and the nimonol against some important human pathogens," Brazilian Journal of Microbiology, vol. 42, no. 3, pp. 1007-1016, 2011.

[29] M. Bordoloi, B. Saikia, R. K. Mathur, and B. N. Goswami, "A meliacin from Chisocheton paniculatus," Phytochemistry, vol. 34, no. 2, pp. 583-584, 1993.

[30] V. Sharma, S. Walia, J. Kumar, M. G. Nair, and B. S. Parmar, "An efficient method for the purification and characterization of nematicidal azadirachtins A, B, and $\mathrm{H}$, using MPLC and ESIMS," Journal of Agricultural and Food Chemistry, vol. 51, no. 14, pp. 3966-3972, 2003. 